\title{
Ionisationsquerschnitt von OV gegenüber Elektronenstoß unter teilweiser Berücksichtigung des Austauschs
}

\author{
Von F. B. Malik * und E. Trefftz \\ Aus dem Max-Planck-Institut für Physik und Astrophysik, München \\ (Z. Naturforschg. 16 a, 583-598 [1961] ; eingegangen am 23. Dezember 1960)
}

\begin{abstract}
The ionization cross-section of highly ionized oxygen, $\mathrm{O}^{4+}$, is calculated according to the "distorted-wave" method. Exchange between the scattered and the ejected electron is taken into account as far as it is of long range nature. It is shown that contributions of high total angular momentum $L$ are essential, $L=0$ giving only $3 \%$ of the total cross-section. This result should qualitatively be the same for all highly ionized atoms, whereas the following seems to be a special feature of $\mathrm{O} \mathrm{V}$ ionization: for energies around twice the ionization energy the contributions of the optically allowed transitions of the ejected electron (angular momentum $l_{\mathrm{ej}}=1$ ) are relatively small. The contributions of $l_{\mathrm{ej}}=0,1,2$ and 3 are about $16 \%, 18 \%, 24 \%$ and $19 \%$ respectively for $E=20 \cdot 13.6 \mathrm{eV}=2.39$ $\times$ Ionization energy. The maximum cross section is 0.112 at. $\mathrm{u} .=0.31 \cdot 10^{-18} \mathrm{~cm}^{2}$ for electrons of $310 \mathrm{eV}$ kinetic energy $(2.8 \times$ ionization energy $)$. It is about twice as large as given by the ElwerT formula.
\end{abstract}

Viele Beobachtungen astrophysikalischer Natur lassen sich unter der Annahme thermodynamischen Gleichgewichts nicht erklären, so z. B. die Emission der Sonnenkorona ${ }^{1}$, die der planetarischen Nebel ${ }^{2}$, die als leuchtende Wolken heiße Sterne umgeben, wie auch Erscheinungen der oberen Erdatmosphäre ${ }^{3}$. Auch die Physik der Gasentladungen und die Erscheinungen, die die Versuche zur Erzielung extrem hoher Temperaturen begleiten ${ }^{4}$, lassen sich nur verstehen über eine detaillierte Betrachtung der möglichen Einzelvorgänge. $\mathrm{Zu}$ dem Zweck braucht man die Kenntnis von Wirkungsquerschnitten sowohl gegenüber Photonen als auch gegenüber Elektronen und schwereren Teilchen. Uns sollen hier Elektronenstoßquerschnitte interessieren für unelastische Stöße, bei denen das getroffene Atom angeregt oder ionisiert wird.

In einer Arbeit über den Durchgang schneller Korpuskularstrahlen durch Materie behandelt BEтHE ${ }^{5}$ das Problem für den Fall, daß die stoßenden und gestreuten Elektronen durch ebene Wellen darstellbar sind. Damit erhält er das asymptotische Verhalten der Querschnitte für unelastische Stöße bei hohen Energien:

$$
Q=\frac{\text { const }}{E} \ln (4 \tau E / h c \mathrm{Ry}),
$$

wobei $E$ die kinetische Energie der einfallenden Elektronen und $\tau$ eine Zahl der Größenordnung 1 ist.

* z. Zt. ICA Fellow with Pakistan Atomic Energy Commission, Palmer Phys. Lab., Princeton (N. J.), USA.

1 L. Biermann, Naturwiss. 34, 87 [1947]. - G. Elwert, Z. Naturforschg. 7 a, 432 [1952]. - A. UnsöLd, Z. Astrophys. 50, 48 [1960] (benutzt Wirkungsquerschnitte von ScHwarz und Ziris ${ }^{8}$, die etwa um einen Faktor 30 zu klein sind).
$\mathrm{Ry}=13,6 \mathrm{eV} / \mathrm{h} c$ ist die Rydberg-Konstante. Der lnFaktor ist ein typisch quantentheoretischer Effekt. In der von Tномsом ${ }^{6}$ berechneten klassischen Formel tritt er nicht auf. Wir führen den reduzierten Wirkungsquerschnitt ein:

$$
Q_{\mathrm{red}}=Q \frac{\left(E_{\mathrm{i}} / h c \mathrm{Ry}\right)^{2}}{n \pi a_{0}^{2}} .
$$

$E_{\mathrm{i}}$ ist die zur Anregung oder Ionisation des Atoms nötige Energie, die dem System an kinetischer Energie verloren geht; $n$ ist die Anzahl der gleichwertigen äußeren Atomelektronen, die angeregt bzw. losgeschlagen werden; $\pi a_{0}^{2}=0,88 \cdot 10^{-16} \mathrm{~cm}^{2}$ ist der Querschnitt des Wasserstoffatoms. Nach THomson ist

$$
Q_{\text {red }}=4(U-1) / U^{2}
$$

mit

$$
U=E / E_{\mathrm{i}}
$$

eine universale Funktion. Das liegt daran, daß der Stoß als Zweier-Stoß zwischen den Elektronen aufgefaßt wird. Dabei wird das übrige Atom nur insofern berücksichtigt, als dem gebundenen Elektron eine Mindestenergie $E_{\mathrm{i}}$ übertragen werden muß. Für kleine Energien $E \lesssim 2 E_{\mathrm{i}}$ wurde die klassische Formel von ELwERT $^{7}$ erweitert und an die seinerzeit vorliegenden genaueren Berechnungen und Messungen angepaßt.

$$
Q_{\mathrm{red}}=c_{1} \frac{U-1}{U^{2}}\left[1+c_{2}(U-1)\right]
$$

2 M. J. Seaton, Rep. Progr. Phys. 23, 313 [1960].

3 G.P.Kuiper, The Earth as a Planet, Univ. of Chicago Press 1954.

4 G. Knorr, Z. Naturforschg. 13 a, 940 [1958].

5 H. Bethe, Ann. Phys., Lpz. 5, 325 [1930].

6 J. J. Thомson, Philos. Mag. J. Sci. 23, 449 [1912].

7 G. Elwert, l. c. ${ }^{1}$. 
mit $c_{1}=2, c_{2}=0,3$. Elwert verwendet seine Formel zur Bestimmung der Elektronentemperatur der Sonnenkorona. Dabei ergibt sich, daß die Elektronentemperatur, $\sim 10^{6}{ }^{\circ} \mathrm{K}$, nur etwa halb so hoch wie die aus der Doppler-Breite der Linien folgende Ionentemperatur ist. Diese Diskrepanz erregte Zweifel an der Richtigkeit der Elwertschen Querschnitte schon der Größenordnung nach ${ }^{8}$. So begannen in letzter Zeit intensive Bemühungen um gute, quantentheoretisch fundierte Formeln für Elektronenstoßquerschnitte, bzw. detaillierte Rechnungen einzelner Fälle. Die Ergebnisse sollen im Zusammenhang mit unseren eigenen Ergebnissen diskutiert werden.

Wir haben den Wirkungsquerschnitt für Ionisation von 4-fach ionisiertem Sauerstoff, O V, berechnet nach der sogenannten „distorted wave"-Methode ${ }^{9}$, die unten im einzelnen beschrieben wird. AustauschEffekte zwischen losgeschlagenem und gestreutem Elektron sind berücksichtigt, sofern sie von langer Reichweite sind, sich also über den Atomradius hinaus erstrecken. Leider haben wir in unserem Fall noch nicht die Möglichkeit, die Ergebnisse mit experimentell bestimmten Wirkungsquerschnitten zu vergleichen, da es zur Zeit nur Messungen an neutralen Stoffen gibt.

\section{Methode}

Der Ionisationsquerschnitt von $\mathrm{OV}$ soll für den Fall berechnet werden, daß sich das Ion vor und nach dem Stoß im Grundzustand befindet. Die Elektronenkonfiguration ist

$$
\begin{array}{ll}
\left(1 s^{2}, 2 s^{2}\right)^{1} S+e & \text { vor dem Stoß, } \\
\left(1 s^{2}, 2 s\right)^{2} S+2 \text { e } & \text { nach dem Stoß. }
\end{array}
$$

Die Erfahrung hat gezeigt, daß Austauscheffekte zwischen den ls-Elektronen und den äußeren Elektronen klein sind. Wenn man sie vernachlässigt, kann man das System als ein System von 3 Elektronen beschreiben, die sich in einem vorgegebenen Potential $V_{0}$ bewegen, das von Kern und 1s-Elektronen gebildet wird.

In nichtrelativistischer Näherung sind Gesamtbahndrehimpuls $L$ und Gesamtspin $S$ sowie die zugehörigen $z$-Komponenten $M_{L}$ und $M_{S}$ gute Quantenzahlen, wobei das Ergebnis von $M_{L}$ und $M_{S}$ nicht ab-

8 S. B. Schwarz u. H. Zirin, Astrophys. J. 130, 384 [1959].

9 Vgl. H.S.W. MASsey, Handbuch der Physik 36 (Atome II), hängt. Wir führen die folgenden Bezeichnungen ein (in atomaren Einheiten) :

$L, M_{L}$ Gesamtbahndrehimpuls und dessen $z$-Komponente,

$S, M_{S} \quad$ Gesamtspin und dessen $z$-Komponente,

$E_{\text {tot }} \quad$ Gesamtenergie des Systems,

$l_{0}, k_{0} \quad$ Drehimpuls und Wellenzahl des einfallenden Elektrons,

$l_{1}, k_{1} \quad$ Drehimpuls und Wellenzahl des gestreuten Elektrons,

$l_{2}, k_{2}$ Drehimpuls und Wellenzahl des losgeschlagenen Elektrons.

Durch das Problem ist vorgegeben:

$$
\begin{aligned}
L & =l_{0}, \quad S=\frac{1}{2} \\
E_{\mathrm{tot}} & =E\left(\mathrm{OV},\left(2 \mathrm{~s}^{2}\right)^{1} \mathrm{~S}\right)+\frac{1}{2}{k_{0}}^{2} \\
& =E\left(\mathrm{OVI},(2 \mathrm{~s})^{2} \mathrm{~S}\right)+\frac{1}{2}\left({k_{1}}^{2}+k_{2}{ }^{2}\right) \\
k_{0}{ }^{2} & =k_{1}{ }^{2}+k_{2}{ }^{2}+2 E_{\mathrm{i}} \\
E_{\mathrm{i}}= & E\left(\mathrm{OVI}(2 \mathrm{~s})^{2} \mathrm{~S}\right)-E\left(0 \mathrm{~V}\left(2 \mathrm{~s}^{2}\right)^{1} \mathrm{~S}\right) \text { ist die Ionisie- }
\end{aligned}
$$
rungsenergie von $\mathrm{O} \mathrm{V}$. - Wir wählen:

$$
M_{\mathrm{S}}=\frac{1}{2} \text {. }
$$

Wir wollen die Formeln für Ionisation ableiten in Analogie zu den Formeln für Anregung. Das losgeschlagene Elektron wird dabei vom gestreuten unterschieden, obwohl das Endergebnis in beiden Elektronen symmetrisch sein muß.

Denken wir uns zunächst, um Normierungsschwierigkeiten zu vermeiden, das kontinuierliche Spektrum des losgeschlagenen Elektrons diskret gemacht durch eine entsprechende Randbedingung, so können wir die Wellenfunktion des Gesamtsystems schreiben:

$$
\begin{gathered}
\Psi(1,2,3)=\sum_{\Gamma} \Psi_{\Gamma}(1,2,3), \\
\Psi_{\Gamma}(1,2,3)=\mathrm{const} \cdot A\left\{\frac{\varphi_{1, \Gamma}\left(r_{1}\right)}{r_{1}} \frac{\varphi_{2, \Gamma}\left(r_{2}\right)}{r_{2}} \frac{f_{\Gamma}\left(r_{3}\right)}{r_{3}}\right. \\
\left.\cdot W_{\Gamma}\left(\vartheta_{1} \varphi_{1}, \vartheta_{2} \varphi_{2}, \vartheta_{3} \varphi_{3}\right) \cdot S_{\Gamma}\left(\sigma_{1}, \sigma_{2}, \sigma_{3}\right)\right\} .
\end{gathered}
$$

$\Gamma$ zählt alle möglichen Konfigurationen mit Gesamtdrehimpuls $L$ durch. $A$ ist der Antisymmetrie-Operator. $\sigma_{i}$ ist die Spin-Koordinate des $i$-ten Elektrons. $W_{\Gamma}$ ist eine normierte Eigenfunktion des Drehimpuls-Operators zum Gesamtdrehimpuls $L$. Für die Spinfunktionen $S_{\Gamma}$ hat man zwei Möglichkeiten je nachdem, ob die Elektronen 1 und 2 zusammen ein Singulett oder ein Triplett bilden.

Theory of Atomic Collisions, p. 285, Springer-Verlag, Berlin 1956. 
$\begin{aligned}(1,2) \text {-Singulett: } & S_{\Gamma}=\frac{1}{\sqrt{2}}\left(\alpha_{1} \beta_{2}-\beta_{1} \alpha_{2}\right) \alpha_{3}, \\ (1,2) \text {-Triplett: } & S_{\Gamma}=\frac{1}{\sqrt{6}}\left(2 \alpha_{1} \alpha_{2} \beta_{3}\right. \\ & \left.\quad-\left(\alpha_{1} \beta_{2}+\beta_{1} \alpha_{2}\right) \alpha_{3}\right) .\end{aligned}$

Dabei entspricht $\alpha_{i}$ positivem, $\beta_{i}$ negativem Spin des $i$-ten Elektrons; $\varphi_{1, \Gamma}(r), \varphi_{2, \Gamma}(r)$ seien normierte Atomwellenfunktionen und mögen einem vollständigen System entstammen, das etwa die Eigenfunktionen des $\mathrm{O}$ V-Spektrums annähert. $f_{\Gamma}$ ist die Wellenfunktion eines freien Elektrons. Es ist nicht nötig, daß $\varphi_{1, \Gamma}$ und $\varphi_{2, \Gamma}$ demselben orthogonalen System entnommen sind. Die Konstante vor dem Antisymmetrie-Operator dient der Normierung bezüglich der gebundenen Zustände

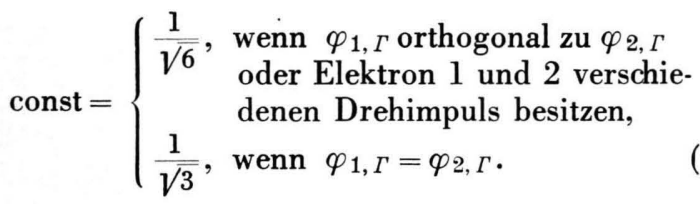

Der Ansatz (7) läßt sich leicht in der Weise erweitern, daß statt des antisymmetrischen Produkts der $\varphi$ bessere 2-Elektronen-Eigenfunktionen des $\mathrm{O}$ V-Spektrums verwendet werden. Die SchröDInger-Gleichung

$$
(H-E) \Psi=0
$$

wird nun in der Weise befriedigt, daß (10) mit je einem Glied der Gestalt

$$
\begin{array}{r}
\Phi_{\Gamma}=\frac{\varphi_{1, \Gamma}\left(r_{1}\right)}{r_{1}} \frac{\varphi_{2, \Gamma}\left(r_{2}\right)}{r_{2}} \\
\cdot W_{\Gamma}\left(\vartheta_{1} \varphi_{1}, \vartheta_{2} \varphi_{2}, \vartheta_{3} \varphi_{3}\right) S_{\Gamma}\left(\sigma_{1}, \sigma_{2}, \sigma_{3}\right)
\end{array}
$$

multipliziert und über die Koordinaten (1), (2) und Winkel und Spin von (3) integriert wird:

$\sum_{\sigma_{1}, \sigma_{3}, \sigma_{3}} \int \ldots \int \Phi_{\Gamma}(H-E) \Psi \mathrm{d}^{3} \mathfrak{r}_{1} \mathrm{~d}^{3} \mathfrak{r}_{2} \mathrm{~d} \Omega_{3}=0$.

Man erhält gewöhnliche gekoppelte Integro-Differentialgleichungen vom HartreE-Fockschen Typ für die $f_{\Gamma}\left(r_{3}\right)$ :

$$
\begin{aligned}
{\left[\frac{\mathrm{d}^{2}}{\mathrm{~d} r^{2}}-\frac{l_{\Gamma}\left(l_{\Gamma}+1\right)}{r^{2}}-2 V_{0}(r)\right.} & \left.-K_{\Gamma \Gamma}(r)+k_{\Gamma}^{2}\right] f_{\Gamma}(r) \\
& =\sum_{\Gamma^{\prime}} K_{\Gamma \Gamma^{\prime}} f_{\Gamma^{\prime}}(r),
\end{aligned}
$$

wobei die $K_{\Gamma \Gamma^{\prime}}$ lineare Integraloperatoren sind:

$$
\begin{aligned}
K_{\Gamma \Gamma^{\prime}}(r) f_{\Gamma^{\prime}}(r)=U_{\Gamma \Gamma^{\prime}}(r) f_{\Gamma^{\prime}}(r) & (14) \\
& +\int K_{\Gamma \Gamma^{\prime}}\left(r, r^{\prime}\right) f_{\Gamma^{\prime}}\left(r^{\prime}\right) \mathrm{d} r^{\prime} .
\end{aligned}
$$

Der multiplikative Anteil $U_{\Gamma \Gamma^{\prime}}(r)$ besteht aus Integralen über die Funktionen $\varphi_{1, \Gamma}, \varphi_{1, \Gamma^{\prime}}, \varphi_{2, \Gamma}, \varphi_{2, \Gamma^{\prime}}$, während in dem Kern $K_{\Gamma \Gamma^{\prime}}\left(r, r^{\prime}\right)$ des Integralopera- tors dieselben Funktionen $\varphi$ als Faktoren auftreten neben Potenzen in $r^{\prime}$ und $r$. Für $\Gamma^{\prime}=\Gamma$ ist $K_{\Gamma \Gamma}(r)$ der HartreE-Fock-Operator; für den einfachen Fall, $\mathrm{da} ß$ Austausch vernachlässigt wird, und nur ein Atomelektron von Interesse ist, ergibt sich

$$
\begin{aligned}
K_{\Gamma \Gamma^{\prime}}(r)= & U_{\Gamma \Gamma^{\prime}}(r) \\
= & \int \varphi_{\Gamma}\left(r^{\prime}\right) W_{\Gamma}\left(\vartheta^{\prime} \varphi^{\prime}, \vartheta \varphi\right) \cdot \frac{2}{\left|\mathfrak{r}-\mathfrak{r}^{\prime}\right|} \\
& \cdot \varphi_{\Gamma^{\prime}}\left(r^{\prime}\right) W_{\Gamma^{\prime}}\left(\vartheta^{\prime} \varphi^{\prime}, \vartheta \varphi\right) \mathrm{d} r^{\prime} \mathrm{d} \Omega^{\prime} \mathrm{d} \Omega \\
= & \sum_{\lambda} c_{\lambda} \cdot 2 \int \varphi_{\Gamma}\left(r^{\prime}\right) \frac{r_{<}^{\lambda}}{r_{>}^{\lambda+1}} \varphi_{\Gamma^{\prime}}\left(r^{\prime}\right) \mathrm{d} r^{\prime},
\end{aligned}
$$

wobei die Summe über $\lambda$ endlich viele Glieder hat entsprechend den nicht verschwindenden Integralen über die Kugelfunktionen in $W_{\Gamma}, W_{\Gamma^{\prime}}$. Der Koeffizient $c_{\lambda}$ ergibt sich aus der Integration über die Winkel; $r_{<}, r_{>}$ist der kleinere bzw. größere der beiden Radien $r, r^{\prime}$. Für zwei Atomelektronen, insbesondere wenn Austausch berücksichtigt wird, werden die Formeln kompliziert. Der Ausdruck für den Wirkungsquerschnitt ist jedoch - nach einigen Vernachlässigungen im Austausch - wieder übersichtlich, so daß wir erst dort die einzelnen Terme besprechen wollen.

Wir wollen in der Summe (6) das Glied, das den Zustand des Systems vor dem Stoß beschreibt, mit dem Index $\Gamma=0$ bezeichnen. In der Distorted-WaveMethode wird angenommen, daß dieses Glied groß ist gegen alle anderen. Unter Berücksichtigung der Form (7) heißt das, daß die Amplitude der Funktion $f_{\Gamma=0}(r)$ für $r \rightarrow \infty$ groß sein soll gegenüber den Amplituden der übrigen $f_{\Gamma}(r)(\Gamma \neq 0)$. Dementsprechend werden die Wechselwirkungen der Glieder mit $\Gamma \neq 0$ untereinander wie auch die Wirkung dieser Glieder auf das $(\Gamma=0)$-Glied vernachlässigt. Damit werden die verschiedenen Konfigurationen $\Gamma \neq 0$ voneinander unabhängig. Um den Wirkungsquerschnitt für einen bestimmten Zustand nach dem Stoß $\mathrm{zu}$ berechnen, braucht man in (6) nur die beiden Terme $\Gamma=0$ und $T=1$ aufzuschreiben, die dem $\mathrm{Zu}$ stand vor dem Stoß und diesem speziellen Zustand nach dem Stoß entsprechen. Aus (12) erhält man zwei Gleichungen der Form (13) für die Funktionen $f_{0}(r)$ und $f_{1}(r)$, wobei man in der Gleichung für $f_{0}(r)$ die von $f_{1}(r)$ abhängigen Terme auf der rechten Seite streicht.

Beim Ausintegrieren von (12) macht man sich zunutze, daß die antisymmetrischen Produkte der $\varphi$ Näherungslösungen des $\mathrm{O}$ V-Problems sein sollen. Will man Anregungsquerschnitte berechnen, so ist es konsequent, so zu rechnen, als ob sie wahre Lösun- 
gen wären. Sollen jedoch die Hartree-Fockschen Gleichungen mit Austausch für Ionisation aufgestellt werden, so ist $\varphi_{2, \Gamma=1}(r)$ die Wellenfunktion eines freien Elektrons ${ }^{10}$ mit der Wellenzahl $k_{2}$ und dem Drehimpuls $l_{2}$. Dann muß man davon ausgehen, daß $\varphi_{1, \Gamma=1}(r)$ die wahre Lösung des O VI-Problems darstellt. Denn wegen der Ununterscheidbarkeit des losgeschlagenen Elektrons vom gestreuten ist es gleichgültig, welchem der beiden Elektronen man die Werte $k_{1}, l_{1}$ bzw. $k_{2}, l_{2}$ zuordnet. Die Formeln müssen symmetrisch sein gegen Vertauschung von $k_{1}, l_{1}$ mit $k_{2}, l_{2}$. Das ist nur gewährleistet, wenn der homogene Teil der Gleichung für $f_{T=1}(r)$ formal übereinstimmt mit der Gleichung für $f_{2, T=1}(r)$. Ist diese Symmetrie verletzt, so ist keine Gewähr dafür, daß die Berücksichtigung des Austauschs eine Verbesserung gegenüber Rechnungen ohne Austausch bedeutet ${ }^{11}$.

Wir wollen die Austausch-Gleichungen nicht explizit hinschreiben, sondern die Gleichungen in der Form angeben, wie sie für die numerischen Ergebnisse benutzt wurden. Zunächst wurde der Austausch gestrichen in den homogenen Gliedern, d. h. auf der linken Seite von (13) in $K_{\Gamma T}$. Wir erhalten für die zu (13) gehörigen homogenen Gleichungen, deren Lösungen wir mit $\chi_{\Gamma}(r)$ bezeichnen wollen:

$$
\Gamma=0: \quad l_{0}=L,
$$

$\varphi_{1,0}(r)=\varphi_{2,0}(r)=\varphi_{0}(r)=2$ s-Funktion von $\mathrm{OV}$,

$$
\begin{array}{r}
\mathfrak{Q}\left(\mathrm{OV} ; k_{0}, l_{0}\right) \chi_{0}(r) \equiv\left\{\frac{\mathrm{d}^{2}}{\mathrm{~d} r^{2}}-\frac{l_{0}\left(l_{0}+1\right)}{r^{2}} \quad(16 \mathrm{a})\right. \\
\left.-2 V_{0}(r)-4 \int_{0}^{\infty} \frac{\varphi_{0}{ }^{2}\left(r^{\prime}\right)}{r_{>}} \mathrm{d} r^{\prime}+k_{0}{ }^{2}\right\} \\
\chi_{0}(r)=0 .
\end{array}
$$

$\Gamma=1: \quad \varphi_{1,1}(r)=\varphi_{1}(r)=2$ s-Funktion von $\mathrm{OVI}$,

$$
\begin{array}{r}
\mathfrak{L}\left(\mathrm{OVI} ; k_{1}, l_{1}\right) \chi_{1}(r) \equiv\left\{\frac{\mathrm{d}^{2}}{\mathrm{~d} r^{2}}-\frac{l_{1}\left(l_{1}+1\right)}{r^{2}} \quad(16 \mathrm{~b})\right. \\
\left.-2 V_{0}(r)-2 \int_{0}^{\infty} \frac{\varphi_{1}{ }^{2}\left(r^{\prime}\right)}{r_{>}} \mathrm{d} r^{\prime}+k_{1}{ }^{2}\right\} \chi_{1}(r)=0 .
\end{array}
$$

( $r>$ größerer der beiden Radien $\left.r, r^{\prime}\right)$.

Das sind die Hartree-Gleichungen für ein Elektron im Felde eines O V- bzw. O VI-Ions. Für die

10 Wir numerieren die Funktionen $\varphi_{1, \Gamma=1}, \varphi_{2, \Gamma=1}$ so, daß $\varphi_{1, \Gamma=1}$ das gebundene $2 \mathrm{~s}$-Elektron darstellt.

11 In der Bons-Näherung bzw. der Bons-Coulomb-Näherung werden die beiden Elektronen verschieden behandelt. Eine entsprechende Unterscheidung könnte hier gemacht werden
Funktionen $f_{0}(r), f_{1}(r)$ gelten folgende Gleichungen:

$$
\begin{aligned}
& \mathfrak{Q}\left(\mathrm{OV} ; k_{0}, l_{0}\right) f_{0}(r)=0, \\
& \mathfrak{Q}\left(\mathrm{OVI} ; k_{1}, l_{1}\right) f_{1}(r)=K_{10}(r) f_{0}(r),
\end{aligned}
$$

wobei $K_{10}(r)$ ein Integraloperator ist, dessen genaue Form unten diskutiert wird. Die Randbedingungen sind

$$
\lim _{r \rightarrow 0} f_{\Gamma}(r)=0
$$

und für große $r$

$$
\begin{gathered}
f_{\Gamma}(r) \sim k_{\Gamma}^{-1 / 2}\left[\delta_{\Gamma, 0} \sin \left(k_{\Gamma} r+\frac{Z_{\infty} \Gamma}{k_{\Gamma}} \ln k_{\Gamma} r\right)\right. \\
\left.-\frac{1}{2 i} T_{\Gamma} \exp \left(i k_{\Gamma} r+i \frac{Z_{\infty}}{k_{\Gamma}} \ln k_{\Gamma} r\right)\right], \\
\text { wobei } \quad Z_{\infty \Gamma}=\left\{\begin{array}{lll}
4 & \text { für } & \Gamma=0 \\
5 & \text { für } & \Gamma=1 .
\end{array}\right.
\end{gathered}
$$

die Ladung des Ions O V bzw. O VI ist. Der Wert $T_{\Gamma}$ bestimmt sich aus den Gln. (17). Verstehen wir unter $\chi_{\Gamma}(r)$ speziell die Lösungen von (16), die für kleine $r$ regulär sind,

$$
\lim _{r \rightarrow 0} \chi_{\Gamma}(r)=0,
$$

und für große $r$

$$
\chi_{\Gamma}(r) \sim k_{\Gamma}^{-1 / 2} \sin \left(k_{\Gamma} r+\frac{Z_{\infty} \Gamma}{k_{\Gamma}} \ln k_{\Gamma} r+\alpha_{\Gamma}\right),
$$

so ist

$$
f_{0}(r)=e^{i \alpha_{0}} \chi_{0}(r) \text {. }
$$

$f_{1}(r)$ läßt sich durch zwei linear unabhängige Lösungen von $(16 \mathrm{~b})$ ausdrücken, deren eine $\chi_{1}(r)$ sei. Nimmt man als zweite Lösung $\tilde{\chi}_{1}(r)$ diejenige, die asymptotisch gleich

$$
\tilde{\chi}_{1}(r) \sim k_{1}^{-1 / 2} \exp \left\{i k_{1} r+i \frac{Z_{\infty 1}}{k_{1}} \ln k_{1} r+i \alpha_{1}\right\}
$$

ist, so ergibt sich ${ }^{12}$

$$
\begin{aligned}
& f_{1}(r)=-\chi_{1}(r) \int_{r}^{\infty} \tilde{\chi}_{1} K_{10} f_{0} \mathrm{~d} r^{\prime}-\tilde{\chi}_{1}(r) \int_{0}^{r} \chi_{1} K_{10} f_{0} \mathrm{~d} r^{\prime} \\
& \text { und } \quad T_{1}=2 i e^{i a_{1}} \int_{0}^{\infty} \chi_{1} K_{10} f_{0} \mathrm{~d} r
\end{aligned}
$$

Der Wirkungsquerschnitt $\Delta Q_{L, l_{1}, l_{2}}$ für den speziellen Prozeß, den wir hier betrachten (vorgegebenes

nach der Größe von $k$, z. B. indem man die Wellenfunktion des gestreuten bzw. losgeschlagenen Elektrons noch abhängen läßt von dem Verhältnis $k_{1}: k_{2}$.

12 N. F. Motr u. H. S. W. MAssey, Theory of Atomic Collisions, $2^{\text {nd }}$ ed. Oxford Univ. Press 1949, p. 113. 
$\left.L, S, l_{0}, k_{0}, l_{1}, k_{1}, l_{2}, k_{2}\right)$ ist proportional $\left|T_{1}\right|^{2}$ :

$$
\begin{aligned}
& \Delta Q_{L, l_{1}, l_{2}}=\frac{\pi}{k_{0}{ }^{2}}(2 L+1)\left|T_{1}\right|^{2}=\frac{\pi}{k_{0}{ }^{2}}(2 L+1) \cdot 4 A^{2} \\
& \text { (s. Anm. }{ }^{13} \text { ) } \\
& \text { mit } \quad A=\int_{0}^{\infty} \chi_{1} K_{10} \chi_{0} \mathrm{~d} r .
\end{aligned}
$$

Gl. (22) läßt erkennen, daß die Voraussetzung der Distorted-Wave-Methode immer dann gut erfüllt ist, wenn $A \ll 1$ ist, oder nach (24) auch, wenn die Stoß-Stärke

$$
\Delta \Omega=\Delta Q k_{0}{ }^{2} / \pi \ll 1 .
$$

In unserem Fall ist der Zustand $\Gamma=1$ ein Kontinuumszustand. In $K_{10}$ tritt deshalb, wie unter Gl. (10) erwähnt, neben $\varphi_{0}(r)$ (2s-Funktion von $O \mathrm{~V}$ ) und $\varphi_{1}(r)$ (2s-Funktion von O VI) auch die Kontinuumsfunktion $\varphi_{2, \Gamma=1}(r)$ auf mit der Wellenzahl $k_{2}$ und dem Drehimpuls $l_{2}$. Konsequenterweise muß sie in derselben Näherung berechnet werden wie $\chi_{1}$, nämlich unter Vernachlässigung des Austauschs mit den gebundenen Elektronen. Es gilt für sie Gl. (16 b), wenn man $k_{1}, l_{1}$ durch $k_{2}, l_{2}$ ersetzt. Die reguläre Lösung dieser Gleichung mit denselben Randbedingungen, die für $\chi_{1}$ gelten, nennen wir $\chi_{2}$. Die in der $k^{2}$-Skala normierte Wellenfunktion ist dann

$$
\varphi_{2, \Gamma=1}(r)=\frac{1}{\sqrt{\pi}} \chi_{2}(r)
$$

mit $\int_{0}^{\infty} \varphi_{2,1}\left(k^{\prime}, r\right) \varphi_{2,1}\left(k_{2}, r\right) \mathrm{d} r=\delta\left(k^{\prime 2}-k_{2}^{2}\right)$.

$$
\text { Das Integral } \quad A=\int_{0}^{\infty} \chi_{1} K_{10} \chi_{0} \mathrm{~d} r
$$

besteht aus einer Anzahl von Gliedern, die wir in zwei Sorten einteilen können, je nachdem, ob der Integrand des Integrals außerhalb des Atoms verschwindet oder nicht. Ein Beispiel der ersten Sorte ist

$$
\begin{aligned}
& \text { const } \cdot \int_{0}^{\infty} \varphi_{0} \chi_{1} \mathrm{~d} r \cdot \int_{0}^{\infty} \varphi_{1}\left(r_{1}\right) \chi_{2}\left(r_{2}\right) \cdot \frac{r_{<}^{L}}{r_{>}^{L+1}} \\
& \cdot \chi_{0}\left(r_{1}\right) \varphi_{0}\left(r_{2}\right) \mathrm{d} r_{1} \mathrm{~d} r_{2}
\end{aligned}
$$

( $r<$ kleinerer, $r_{>}$größerer der beiden Radien $r_{1}, r_{2}$ ). Der Integrand ist nur innerhalb des Atoms von Null verschieden. Von ähnlicher Gestalt sind auch die

13 Im allgemeinen steht im Nenner der rechten Seite von (24) noch das statistische Gewicht des Ausgangszustandes des Atoms, das bei uns gleich 1 ist. Die Normierung von $\chi_{0}, \chi_{1}$
Glieder, die wir in der Differentialgleichung weggelassen haben. Deshalb sollen diese Glieder auch hier vernachlässigt werden. Beibehalten werden sollen nur solche Glieder, deren Integrand sich über das Atom hinaus erstreckt. Es treten folgende Integrale auf

$$
\begin{gathered}
V_{1}=\left(\varphi_{0}, \varphi_{1}\right) \cdot c_{l_{2} l_{1}}^{L} \int_{0}^{\infty} \chi_{1}\left(r_{1}\right) \chi_{2}\left(r_{2}\right) \frac{2 r_{>}^{l_{2}}}{r_{>}+1} \\
\cdot \chi_{0}\left(r_{1}\right) \varphi_{0}\left(r_{2}\right) \mathrm{d} r_{1} \mathrm{~d} r_{2}, \\
V_{2}=\left(\varphi_{0}, \varphi_{1}\right) \cdot c_{l_{1} l_{2}}^{L} \int_{0}^{\infty} \chi_{2}\left(r_{1}\right) \chi_{1}\left(r_{2}\right) \frac{2 r_{>}^{l_{1}}}{r_{>}^{l_{1}+1}} \\
\cdot \chi_{0}\left(r_{1}\right) \varphi_{0}\left(r_{2}\right) \mathrm{d} r_{1} \mathrm{~d} r_{2}, \\
V_{3}=\delta_{l_{1} 0} \int_{0}^{\infty} \varphi_{0} \chi_{1} \mathrm{~d} r \int_{0}^{\infty} \chi_{2}\left(r_{1}\right)\left(-2\left(\varphi_{0}, \varphi_{1}\right) \varphi_{0}\left(r_{2}\right)\right. \\
\left.+\varphi_{1}\left(r_{2}\right)\right) \frac{2}{r_{>}} \chi_{0}\left(r_{1}\right) \varphi_{0}\left(r_{2}\right) \mathrm{d} r_{1} \mathrm{~d} r_{2}, \\
V_{4}=\delta_{l_{2} 0} \int_{0}^{\infty} \varphi_{0} \chi_{2} \mathrm{~d} r \int_{0}^{\infty} \chi_{1}\left(r_{1}\right)\left(-2\left(\varphi_{0}, \varphi_{1}\right) \varphi_{0}\left(r_{2}\right)\right. \\
\left.+\varphi_{1}\left(r_{2}\right)\right) \frac{2}{r_{>}} \chi_{0}\left(r_{1}\right) \varphi_{0}\left(r_{2}\right) \mathrm{d} r_{1} \mathrm{~d} r_{2},
\end{gathered}
$$

mit

$$
\left(\varphi_{0}, \varphi_{1}\right)=\int_{0}^{\infty} \varphi_{0}(r) \varphi_{1}(r) \mathrm{d} r
$$

$V_{2}$ geht aus $V_{1}$, und $V_{4}$ aus $V_{3}$ hervor durch Vertauschung des losgeschlagenen mit dem gestreuten Elektron.

$$
c_{l_{2} l_{1}}^{L} \underset{r_{>} l_{2}+1}{l_{2}^{l_{2}}}=\left\langle\left(l_{1}(1), l_{2}(2)\right) L\left|\frac{1}{r_{12}}\right|(L(1), S(2)) L\right\rangle
$$

folgt aus der Integration der Wellenfunktion über die Winkel $\left(l_{1}(1), l_{2}(2)\right) L$ steht für eine Eigenfunktion des Drehimpulsoperators zum Gesamtdrehimpuls $L$, mit den angegebenen Drehimpulsen der einzelnen Elektronen. Die Formel für $c_{l_{2}}^{L} l_{1}$ haben wir aus Condon-Shortley ${ }^{14}$ entnommen. Sie ist im Abschnitt Rechentechnik unter a) angegeben. Entsprechend unserer Ableitung ergeben sich in A, Gl. (25) verschiedene Koeffizienten, je nachdem, ob das gebundene und das losgeschlagene Elektron zusammen ein Singulett- oder ein Triplett-System bilden:

$$
A=\sqrt{2} \frac{1}{\sqrt{\pi}}\left[\left\{\begin{array}{l}
1 \\
0
\end{array}\right\}\left(V_{1}+V_{4}\right)-\left\{\begin{array}{c}
1 / 2 \\
7^{3 / 2}
\end{array}\right\}\left(V_{2}+V_{3}\right)\right] .
$$

ist so gewählt, daß außer dem Faktor $1 / k_{0}{ }^{2}$ keine weiteren Faktoren $k_{0}, k_{1}$ in (24) auftreten.

14 E. U. Condon u. G. H. Shortley, Theory of Atomic Spectra, Cambridge 1951. 
Die obere Zahl in der geschweiften Klammer entspricht der Singulett-Anregung, die untere der Triplett-Anregung. Der Faktor $\sqrt{2}$ kommt dadurch herein, daß die Ionisation aus einer Schale mit zwei äquivalenten Elektronen erfolgt. Er ergibt sich formal aus dem Verhältnis der beiden Normierungskonstanten der Wellenfunktionen vor und nach dem Stoß, gemäß Gl. (7) und (9). Durch den Faktor $1 / \sqrt{\pi}$ wird die Wellenfunktion des losgeschlagenen Elektrons in der $k^{2}$-Skala normiert. Das bedeutet, $\mathrm{da} ß$ wir, wenn wir den Ausdruck (31) für $A$ in Gl. (24) einsetzen, den Wirkungsquerschnitt pro $k^{2}$ Intervall erhalten. $V_{4}$ ist eine kleine Korrektur zu $V_{1}$ in dem Fall, daß ein s-Elektron losgeschlagen wird, $l_{2}=0$. Sie rührt daher, daß hier nicht angenommen ist, daß $\varphi_{1, \Gamma=1}, \varphi_{2, \Gamma=1}$ unter Berücksichtigung der Symmetrisierung eine Lösung des O V-Kontinuums darstellen, sondern nur daß $\varphi_{1, \Gamma=1}=\varphi_{1}$ den $\mathrm{O}$ VIGrundzustand gibt. Entsprechend tritt $V_{3}$ als Korrektur zu $V_{2}$ auf für $l_{1}=0$, wenn man die Rolle von gestreutem und losgeschlagenem Elektron vertauscht.

Summation von Singulett- und Triplett-Anregung ergibt:

$$
\begin{aligned}
& \frac{\mathrm{d} Q_{L, l_{1}, l_{2}}}{\mathrm{~d}\left(k_{2}{ }^{2}\right)}=\frac{4 \pi}{k_{0}^{2}}(2 L+1)\left(A_{\text {sing }}^{2}+A_{\text {trip }}^{2}\right) \\
& =\frac{8}{k_{0}^{2}}(2 L+1)\left[\left(V_{1}+V_{4}\right)^{2}+\left(V_{2}+V_{3}\right)^{2}\right. \\
& \left.-\left(V_{1}+V_{4}\right)\left(V_{2}+V_{3}\right)\right] .
\end{aligned}
$$

Die Formel hat die gewünschte Symmetrie zwischen gestreutem und losgeschlagenem Elektron. Die ersten zwei Glieder treten ebenso auf wie in der Rechnung ohne Austausch, wo sie zwei verschiedene Prozesse beschreiben:

$$
\begin{array}{ll}
\left(V_{1}+V_{4}\right)^{2}: & \begin{array}{l}
\text { gestreutes Elektron mit } k_{1}, l_{1}, \\
\text { losgeschlagenes Elektron mit } k_{2}, l_{2}, \\
\left(V_{2}+V_{3}\right)^{2}:
\end{array} \\
\text { gestreutes Elektron mit } k_{2}, l_{2}, \\
\text { losgeschlagenes Elektron mit } k_{1}, l_{1} .
\end{array}
$$

Das letzte ist ein zusätzliches Austauschglied.

Um den Gesamtwirkungsquerschnitt zu erhalten, muß zunächst über $k_{2}^{2}$ integriert werden:

$$
Q_{L, l_{1}, l_{2}}=\int_{0}^{k_{0}{ }^{2}-2 E_{1}} \frac{\mathrm{d} Q_{L, l_{1}, l_{2}}}{\mathrm{~d} k_{2}{ }^{2}} \mathrm{~d}\left(k_{2}{ }^{2}\right),
$$

und über alle möglichen Tripel $L, l_{1}, l_{2}$ summiert

15 W. Elsasser, Z. Phys. 45, 522 [1927]. - N. F. Mott u. H. S. W. Massey, Theory of Atomic Collisions, $2^{\text {nd }}$ ed. Oxford 1949, p. 241. werden:

$$
Q_{L}=\sum_{l_{1}, l_{2}} Q_{L, l_{1}, l_{2}}
$$

mit den Bedingungen

$$
\begin{aligned}
& L+l_{1}+l_{2} \quad \text { gerade, } \\
& \left|L-l_{1}\right| \leq l_{2} \leq L+l_{1} \quad \text { (Dreiecksbedingung) }
\end{aligned}
$$

und

$$
Q_{\text {gesamt }}=Q\left(k_{0}{ }^{2}\right)=\sum_{L=0}^{\infty} Q_{L} .
$$

Die Durchführung der Rechnung wird im Abschnitt Rechentechnik beschrieben.

\section{Ergebnisse}

Berechnet wurden die Energiewerte $k_{0}{ }^{2}=9 ; 12$; 20 at. E., das entspricht $E=1,075 E_{\mathrm{i}} ; 1,433 E_{\mathrm{i}}$; $2,389 E_{\mathrm{i}}\left(E_{\mathrm{i}}=8,37212 h c \mathrm{Ry}\right)$. Höhere Energien konnten leider nicht erfaßt werden, da dafür der Drehimpulsbereich $l_{1}, l_{2} \leq 5$ zu klein gewählt war. Sie sollen bei nächster Gelegenheit nachgetragen werden. Die in den Tabellen in eckigen Klammern gegebenen Zahlen sind geschätzt.

Um den Vergleich mit anderen Arbeiten zu erleichtern, betrachten wir zunächst die Nichtaustauschglieder

$$
\frac{8(2 L+1)}{k_{0}^{2}} \int_{0}^{k_{0}^{2}-2 E_{1}}\left(V_{1}+V_{4}\right)^{2} \mathrm{~d}\left(k_{2}{ }^{2}\right),
$$

aufgegliedert nach Gesamtdrehimpuls $L$ und Drehimpuls des losgeschlagenen Elektrons $l_{\mathrm{ej}}=l_{2}$, Tab. 1 . Für hohe Energien sollten die Terme mit $l_{2}=1$, die den optischen UUbergängen des Atoms entsprechen, überwiegen. Sie sind es nämlich, die das logarithmische Verhalten von $Q$, Gl. (1), bewirken, während alle anderen Beiträge mit wachsender Energie wie $1 / E$ abfallen ${ }^{15}$. In unserem Bereich sind die Beiträge höherer $l_{2}\left(l_{2} \geq 2\right)$ erstaunlich groß (vgl. Abb. 1). Der Gesamtbeitrag der Terme mit $l_{2}=1$ zum Wirkungsquerschnitt ist bei $k_{0}{ }^{2}=20$ etwas höher als bei $k_{0}^{2}=12$, bleibt aber unter $20 \%$, während in den Fällen, die SEAton und Mitarbeiter ${ }^{16}$ gerechnet haben, etwa 60 bis $70 \%$ auf optische Übergänge entfallen. Vermutlich liegt die Ursache darin, daß die optischen Übergänge vom Grundzustand ins Kontinuum verhältnismäßig schwach sind ${ }^{17}$.

16 A. Burgess, Astrophys. J. 132, 503 [1960].

17 Für diese Bemerkung sind wir Herrn Dr. Seaton zu Dank verpflichtet. 


\begin{tabular}{|c|c|c|c|c|c|c|c|c|c|c|c|c|c|}
\hline \multirow{3}{*}{$\begin{array}{l}3 \\
11\end{array}$} & & $\overrightarrow{1}$ & $\begin{array}{l}10 \\
80 \\
80 \\
00\end{array}$ & ôे & \multirow{3}{*}{$\frac{0}{8}$} & & 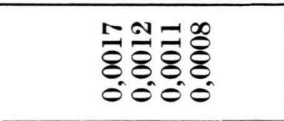 & \begin{tabular}{l}
$\infty$ \\
\multirow{8}{*}{} \\
0 \\
0
\end{tabular} & \multirow{3}{*}{$\begin{array}{l}\text { 象 } \\
\text { : } \\
0 \\
0\end{array}$} & & 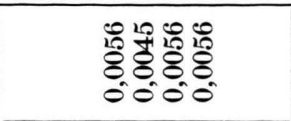 & חั & \multirow{3}{*}{ 栾 } \\
\hline & & $\begin{array}{l}\infty \\
1 \\
4\end{array}$ & $\begin{array}{l}\text { 范 } \\
80 \\
0\end{array}$ & $\begin{array}{l}\text { ¿े } \\
8 \\
0\end{array}$ & & & 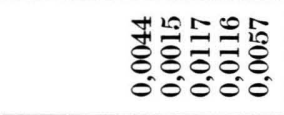 & 粂 & & & 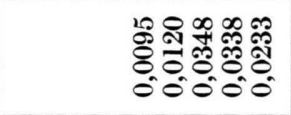 & $\stackrel{\leftrightarrow}{\leftrightarrows}$ & \\
\hline & 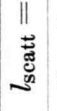 & i & $\begin{array}{l}\text { है } \\
\Xi_{0} \\
0\end{array}$ & 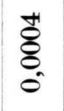 & & & 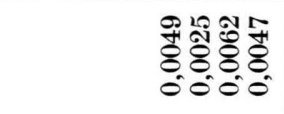 & $\stackrel{\infty}{\infty}$ & & & 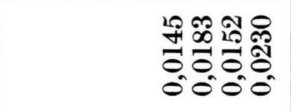 & 옹 & \\
\hline \multirow{3}{*}{$\begin{array}{l}9 \\
4 \\
4\end{array}$} & & 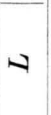 & 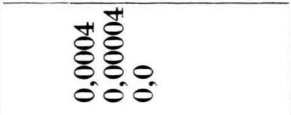 & 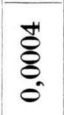 & \multirow{3}{*}{$\begin{array}{l}0 \\
20 \\
0 \\
0\end{array}$} & & 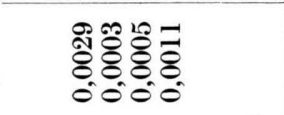 & \begin{tabular}{l}
$\infty$ \\
\multirow{8}{*}{} \\
0
\end{tabular} & \multirow{3}{*}{ 告 } & & 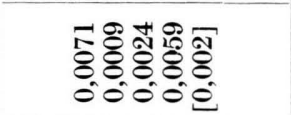 & 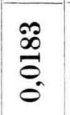 & \multirow{3}{*}{$\begin{array}{l}5 \\
0 \\
\infty \\
0 \\
0\end{array}$} \\
\hline & & a & 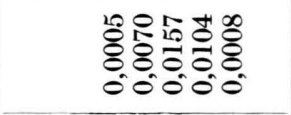 & 薄 & & & 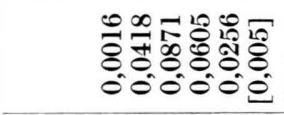 & 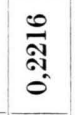 & & & 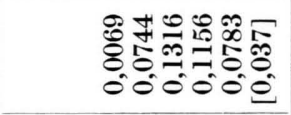 & 畨 & \\
\hline & 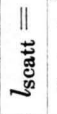 & $\begin{array}{l}4 \\
+ \\
+1\end{array}$ & 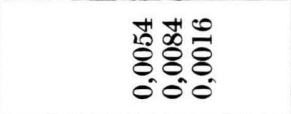 & $\begin{array}{l}\text { 渵 } \\
0 \\
0 \\
0\end{array}$ & & & 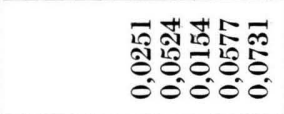 & 㥼 & & & 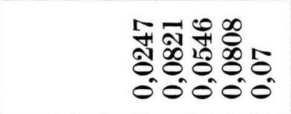 & 胥 & \\
\hline \multirow{4}{*}{ 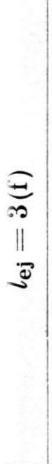 } & & $\begin{array}{l}\infty \\
+ \\
+\end{array}$ & 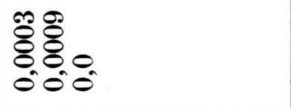 & $\underset{0}{\tilde{8}}$ & \multirow{4}{*}{$\begin{array}{l}\frac{1}{2} \\
\frac{1}{6} \\
0 \\
0\end{array}$} & & 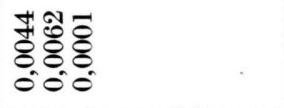 & $\begin{array}{l}5 \\
0 \\
0 \\
0\end{array}$ & \multirow{4}{*}{ ڤั: } & & 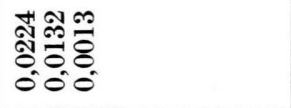 & $\begin{array}{l}\mathscr{8} \\
\mathscr{8} \\
0 \\
0\end{array}$ & \multirow{4}{*}{ 递 } \\
\hline & & $\begin{array}{l}\overrightarrow{1} \\
+ \\
+\end{array}$ & 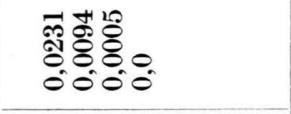 & 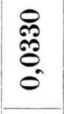 & & & 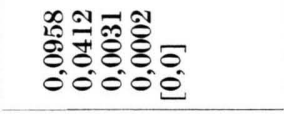 & $\frac{\mathscr{q}}{\stackrel{+}{+}}$ & & & 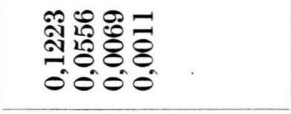 & $\begin{array}{l}0 \\
0 \\
0 \\
0 \\
0\end{array}$ & \\
\hline & & İ & 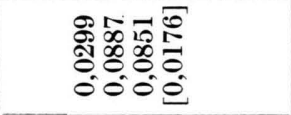 & 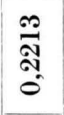 & & & 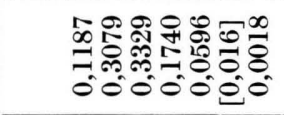 & $\stackrel{8}{0}$ & & & 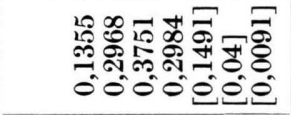 & 焈 & \\
\hline & 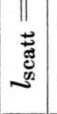 & $\begin{array}{c}\infty \\
1 \\
1 \\
-1\end{array}$ & 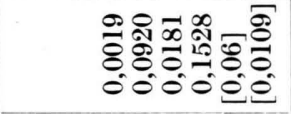 & $\begin{array}{l}5 \\
0 \\
0 \\
0 \\
0\end{array}$ & & & 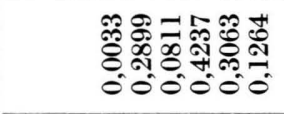 & 今్ & & & 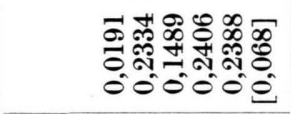 & $\begin{array}{l}\infty \\
\infty \\
\text { S్ } \\
0 \\
0\end{array}$ & \\
\hline \multirow{3}{*}{ 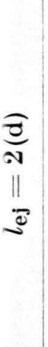 } & & $\begin{array}{l}\text { N } \\
+ \\
+\end{array}$ & 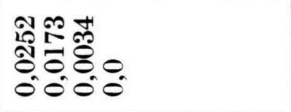 & 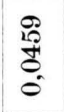 & \multirow{3}{*}{ 年 } & & 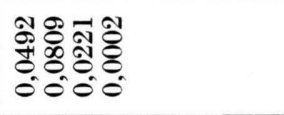 & 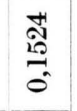 & \multirow{3}{*}{$\underset{7}{F}$} & & 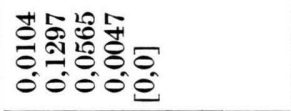 & 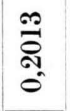 & \multirow{3}{*}{$\begin{array}{l}\text { मे } \\
\ddot{0} \\
0 \\
\infty\end{array}$} \\
\hline & & $\dashv$ & 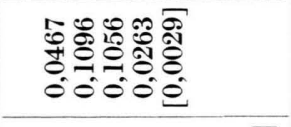 & ] & & & 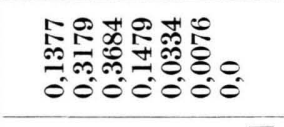 & $\stackrel{\stackrel{9}{0}}{0}$ & & & 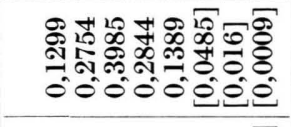 & 骨 & \\
\hline & 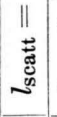 & 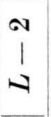 & 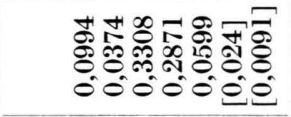 & $\begin{array}{l}5 \\
\text { 点 } \\
\infty \\
0 \\
0\end{array}$ & & & 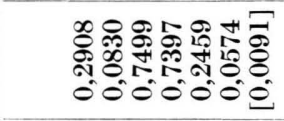 & 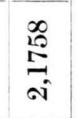 & & & 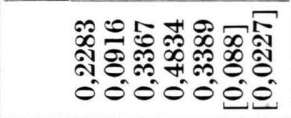 & 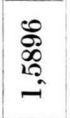 & \\
\hline \multirow{2}{*}{$\begin{array}{l}\widehat{3} \\
11 \\
0\end{array}$} & & $\begin{array}{l}- \\
+ \\
-\end{array}$ & 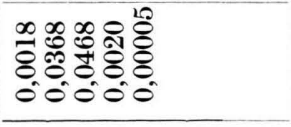 & $\begin{array}{l}0 \\
0 \\
0 \\
0\end{array}$ & \multirow{2}{*}{ 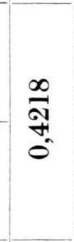 } & & 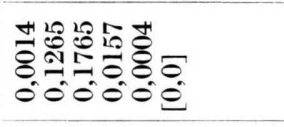 & 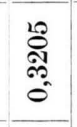 & \multirow{2}{*}{ 商 } & & 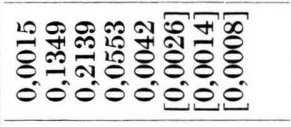 & 栗 & \multirow{2}{*}{$\begin{array}{l}0 \\
\infty \\
\infty \\
\text { oi }\end{array}$} \\
\hline & 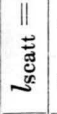 & I & 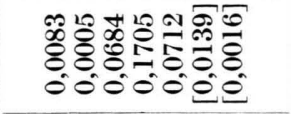 & 范 & & & 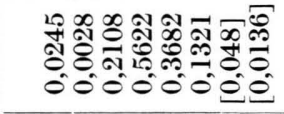 & :ัญ & & & 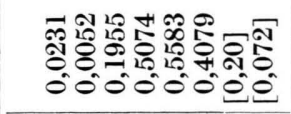 & 苍 & \\
\hline$\overbrace{0}^{\infty}$ & 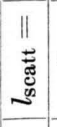 & ヘ & 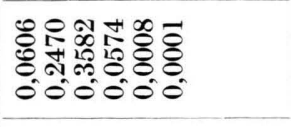 & 方 & F্ & & 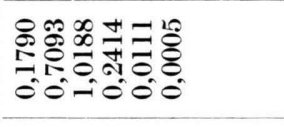 & $\begin{array}{l}\tilde{D} \\
\stackrel{0}{0} \\
\text { ô }\end{array}$ & $\begin{array}{c}\overrightarrow{0} \\
\stackrel{0}{0} \\
\text { ai } \\
\end{array}$ & & 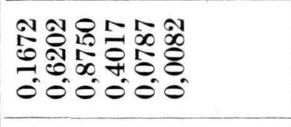 & a) & $\frac{0}{20}$ \\
\hline$\underset{11}{\infty}$ & & ヘ & $0-N \infty+\angle 0 \cap \infty$ & WN & 小憘 & 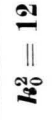 & $0-N \infty+200 \times \infty$ & NA & 小余 & $\begin{array}{l}\text { कิ } \\
\| \\
\text { d॥ㅇ }\end{array}$ & $0-N \infty$ & NA & W苟 \\
\hline
\end{tabular}




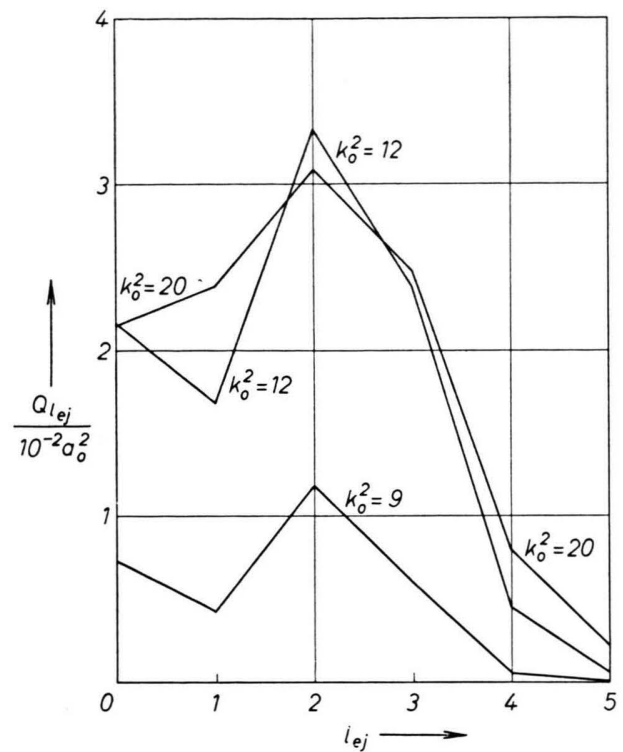

Abb. 1. Anteile der einzelnen Drehimpulse $l_{\mathrm{ej}}$ des losgeschlagenen Elektrons am Gesamtionisationsquerschnitt (ohne Austausch).

Nach Seaton ${ }^{18}$ ist der Photoionisationsquerschnitt an der Schwelle

$$
a_{v_{0}}=0,77 \cdot 10^{-18} \mathrm{~cm}^{2},
$$

während man in Analogie zu wasserstoffähnlichen Spektren etwa $1,7 \cdot 10^{-18} \mathrm{~cm}^{2}$ erwarten sollte. Es würde sich vielleicht lohnen, Ionisationsquerschnitte von Niveaus gleicher Hauptquantenzahl aus zu vergleichen.

In den Abb. 2 a bis c ist

$$
k_{0}{ }^{2} \frac{\mathrm{d} Q}{\mathrm{~d}\left(k_{2}{ }^{2}\right)}=8(2 L+1)\left(V_{1}+V_{4}\right)^{2}
$$

( $Q$ ohne Austausch) über $k_{2}{ }^{2}$ für die wichtigsten Drehimpulstripel $L-l_{\text {scatt }}-l_{\text {ej }}$ aufgetragen [vgl. Gl. (28) mit $\left.l_{\text {scatt }}=l_{1}, l_{\mathrm{ej}}=l_{2}\right]$. Im ganzen bestätigt sich die Bemerkung von Bethe ${ }^{5}$, daß Stöße, bei denen das losgeschlagene Elektron hohe Energie erhält, seltener sind. Beachtlich ist jedoch, daß es bei kleineren Stoßenergien, $\frac{1}{2} k_{0}{ }^{2}<2 E_{\mathrm{i}}$, Drehimpulskombinationen mit $l_{\mathrm{ej}}=2$ oder 3 gibt, die einen wesentlichen Beitrag gerade für großes $k_{2}{ }^{2}(=2 \times$ Energie des losgeschlagenen Elektrons) liefern, vgl. die Kurven $4-2-2$ und $5-2-3$. Bei $k_{0}{ }^{2}=12$ verhalten sich die hier gezeigten Beiträge für $k_{2}^{2}=k_{0}^{2}-2 E_{\mathrm{i}}$ zu denen für $k_{2}{ }^{2}=0$ etwa wie $43: 100$, während bei $k_{0}{ }^{2}=20$ das Verhältnis schon auf $14: 100$ gefallen ist.

18 Persönliche Mitteilung.

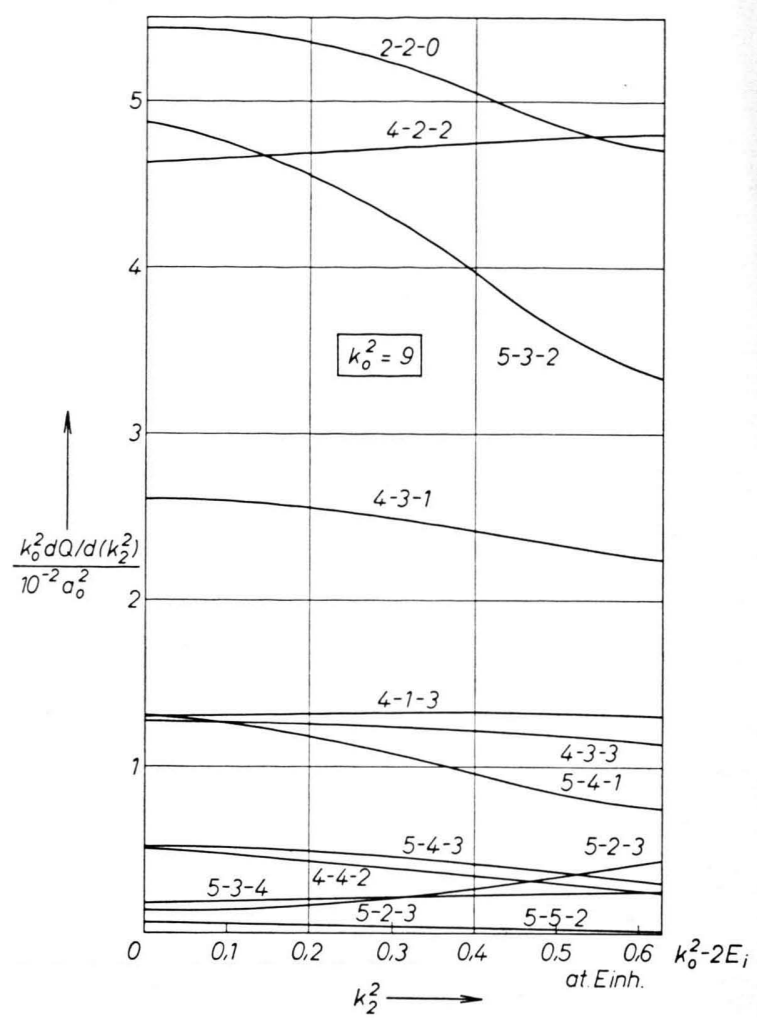

Abb. 2 a.

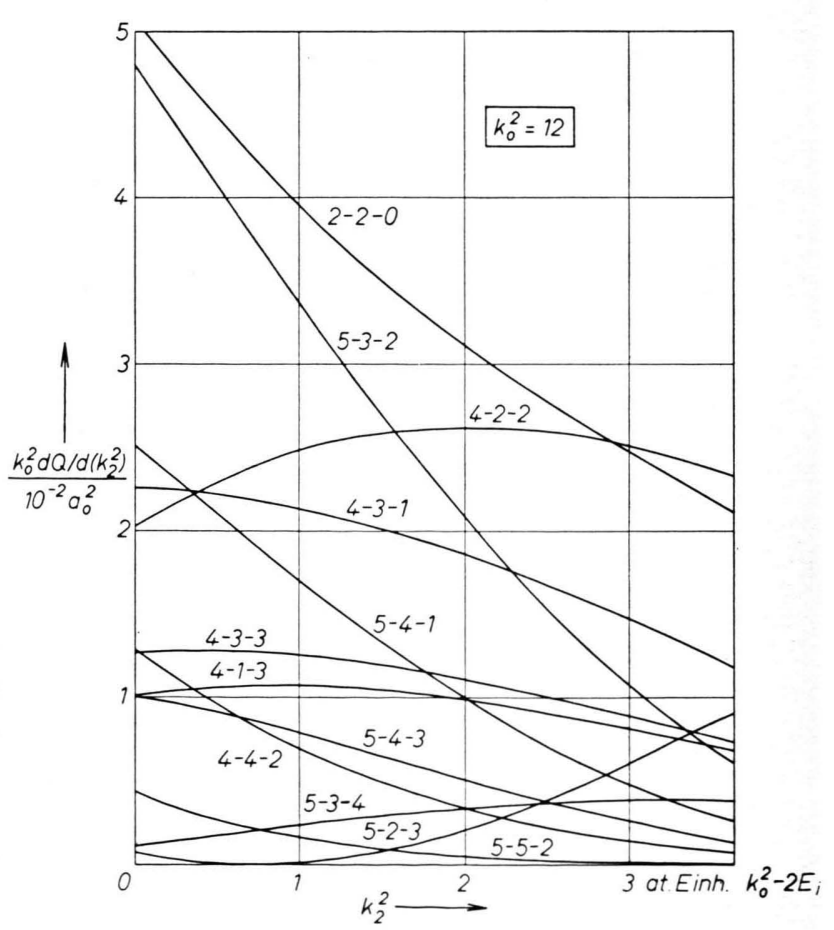

Abb. 2 b. 


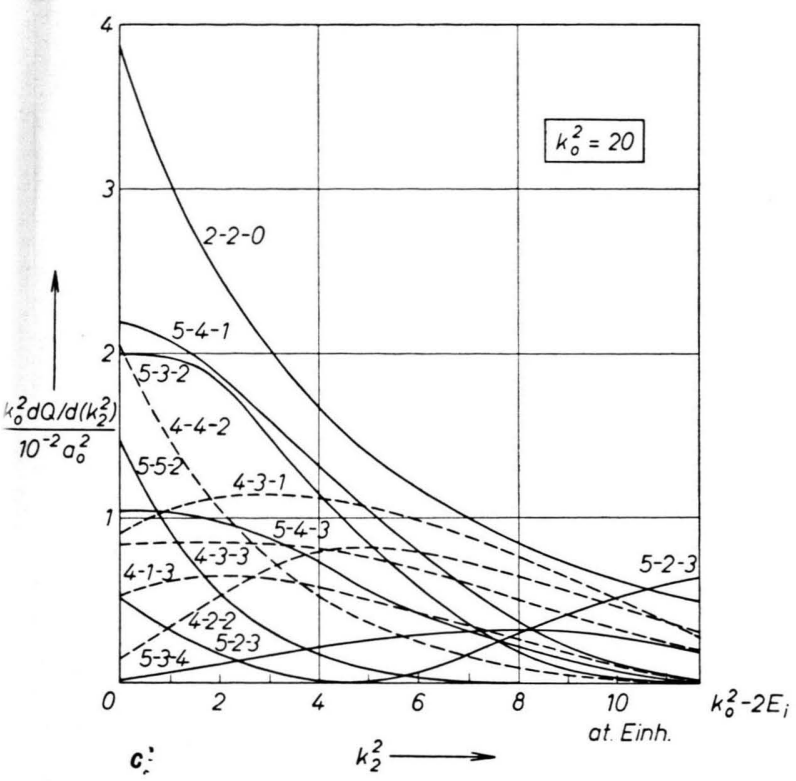

Abb. 2. Verteilung der Beiträge zum Ionisationsquerschnitt über die Energie des losgeschlagenen Elektrons für die wichtigsten Drehimpuls-Tripel $L-l_{\mathrm{scatt}}-l_{\mathrm{ej}}$; a) $k_{0}^{2}=9$; b) $k_{0}^{2}=12$; c) $k_{0}^{2}=20$.

Die Berücksichtigung des Austauschs (s. Tab. 2) gibt den Prozessen $l_{1}=l_{2}$, bei denen gestreutes und losgeschlagenes Elektron gleichen Drehimpuls haben, etwas größere Wahrscheinlichkeit, während für $l_{1} \neq l_{2}$ der Wirkungsquerschnitt im allgemeinen kleiner wird als die Summe der beiden Wirkungsquerschnitte, die in (32) zusammengefaßt sind. [In (32) haben $\left(V_{1}+V_{4}\right)$ im allgemeinen dasselbe Vorzeichen wie $\left(V_{2}+V_{3}\right)$.]

Tab. 3 und Abb. 3 a und $3 \mathrm{~b}$ geben die Beiträge der einzelnen Gesamtdrehimpulse $L$. Sie zeigen ein ziemlich unregelmäßiges Verhalten. Durch den Austausch werden geradzahlige $L$ bevorzugt. Das „Loch“ bei $L=3$ ist wohl ein individueller Auslöscheffekt bei der $\mathrm{O}$ V-Ionisation vom Grundzustand aus. Bemerkenswert ist der große Beitrag für höhere $L$, schon für Energien in der Umgebung der Schwelle. Der Wirkungsquerschnitt für $L=0$ gibt weniger als $3 \%$ des Gesamtwirkungsquerschnitts ${ }^{19}$. Wir hatten eine primitive Abschätzung aus den Größenverhältnissen gemacht: $L$-Werte bis einschließlich $L_{\max }$ sollten berücksichtigt werden, wobei

$$
L_{\max } \approx 2 k \times \text { Atomradius }
$$

19 Da dieses Verhalten bei allen höher geladenen Ionen zu erwarten ist, dürfte der von $\mathrm{Schwarz}_{\text {und }} \mathrm{Z}_{\mathrm{IRIN}}{ }^{8}$ angegebene Ionisationsquerschnitt von Fe XIV, der nur den Beitrag $L=0$ enthält, etwa um einen Faktor 30 zu klein sein. gesetzt wurde. Mit $\quad L_{\max }=8$

( $k \leq 6$; Atomradius $\approx 0,7$ ) hatten wir knapp hoch genug geschätzt. Die einschneidendere Schranke war allerdings, wie bereits erwähnt, die für $l_{1}, l_{2}$, die mit

$$
l_{1}, l_{2} \leq 5
$$
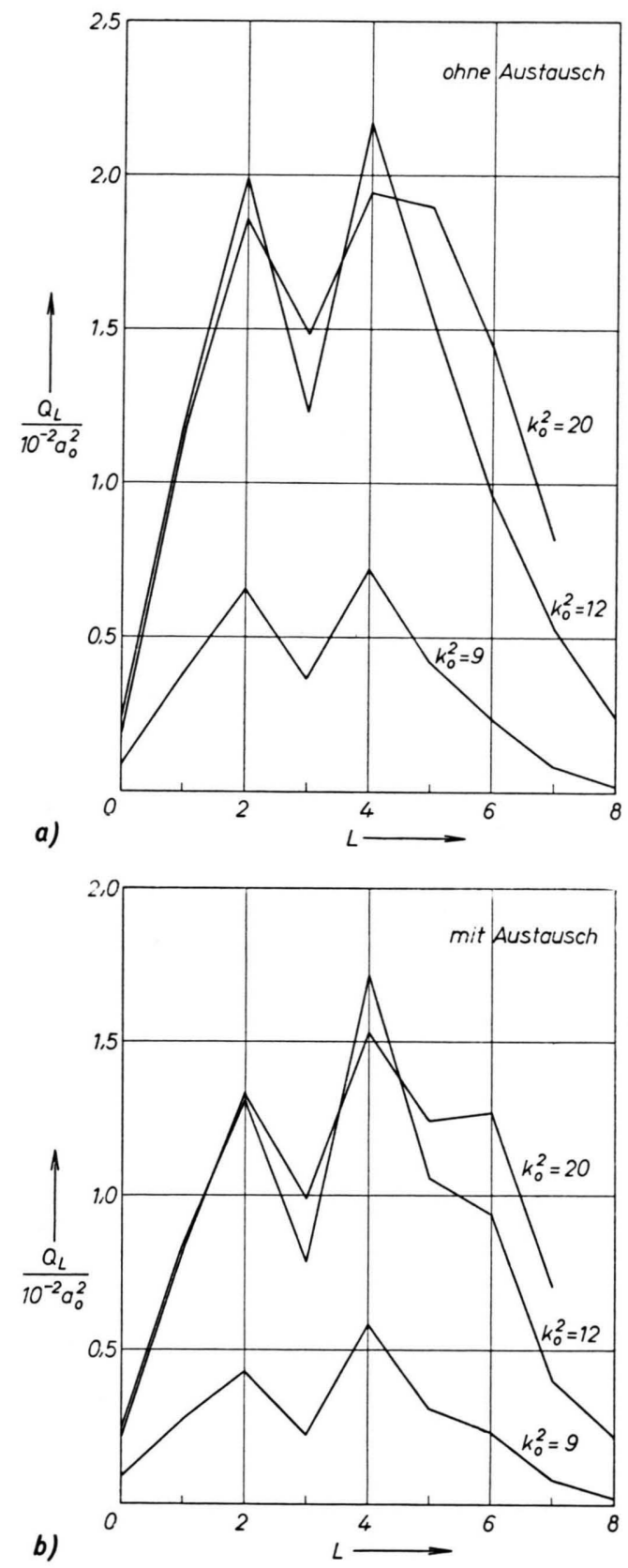

Abb.3. Beiträge der einzelnen Gesamtdrehimpulse $L$ ( (=Drehimpuls des ankommenden Elektrons) zum Ionisationsquerschnitt, a) ohne Austausch; b) mit Austausch großer Reichweite. 


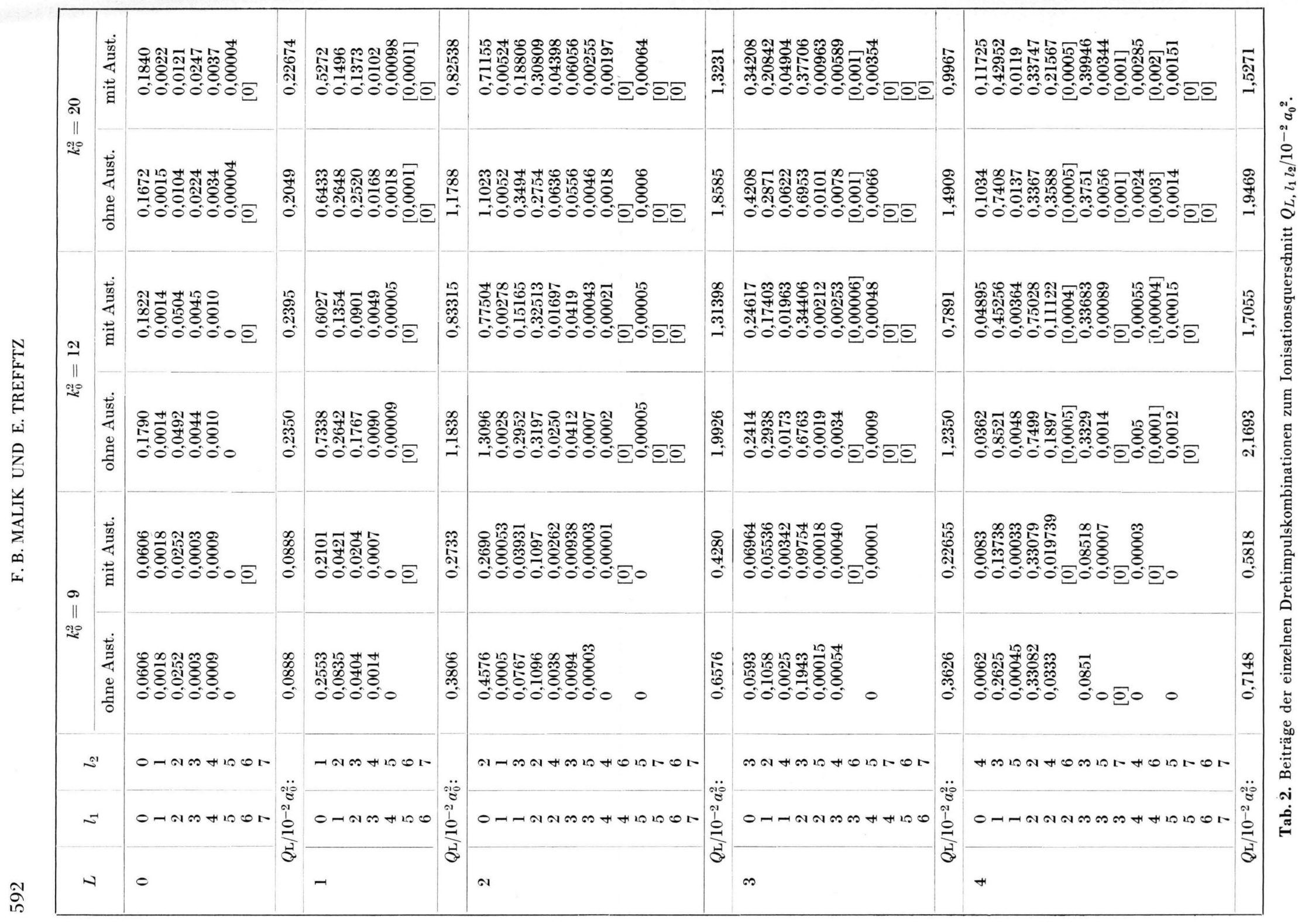




\begin{tabular}{|c|c|c|c|c|c|c|c|c|}
\hline \multirow{2}{*}{$L$} & \multirow{2}{*}{$l_{1}$} & \multirow{2}{*}{$l_{2}$} & \multicolumn{2}{|c|}{$k_{0}^{2}=9$} & \multicolumn{2}{|c|}{$k_{0}^{2}=12$} & \multicolumn{2}{|c|}{$k_{0}^{2}=20$} \\
\hline & & & ohne Aust. & mit Aust. & ohne Aust, & mit Aust. & ohne Aust. & mit Aust. \\
\hline 5 & $\begin{array}{l}0 \\
1 \\
1 \\
2 \\
2 \\
2 \\
3 \\
3 \\
4 \\
4 \\
5 \\
6\end{array}$ & $\begin{array}{l}5 \\
4 \\
6 \\
3 \\
5 \\
7 \\
4 \\
6 \\
5 \\
7 \\
6 \\
7\end{array}$ & $\begin{array}{c}0,00046 \\
0,0796 \\
{[0]} \\
0,3052 \\
{[0,0029]} \\
{[0]} \\
{[0,0333]} \\
{[0]} \\
0\end{array}$ & $\begin{array}{c}0,00027 \\
0,05511 \\
{[0]} \\
0,23364 \\
0,00219 \\
{[0]} \\
0,02442 \\
{[0]} \\
{[0]}\end{array}$ & $\begin{array}{c}0,0005 \\
0,4206 \\
{[0,001]} \\
0,8208 \\
0,0349 \\
{[0]} \\
0,2611 \\
{[0,0002]} \\
0,0022 \\
{[0]} \\
{[0]}\end{array}$ & $\begin{array}{c}0,004308 \\
0,28175 \\
{[0,001]} \\
0,60453 \\
0,02824 \\
{[0]} \\
0,13859 \\
{[0,0002]} \\
0,00109 \\
{[0]} \\
{[0]}\end{array}$ & $\begin{array}{c}0,0227 \\
0,6406 \\
{[0,007]} \\
0,6323 \\
0,1509 \\
{[0,002]} \\
0,4300 \\
{[0,003]} \\
0,0115 \\
{[0]} \\
{[0]}\end{array}$ & $\begin{array}{c}0,02433 \\
0,42919 \\
{[0,005]} \\
0,42234 \\
0,11202 \\
{[0,002]} \\
0,23724 \\
{[0,003]} \\
0,00618 \\
{[0]} \\
{[0]}\end{array}$ \\
\hline \multicolumn{3}{|c|}{$Q \mathrm{~L} / 10^{-2} a_{0}^{2}:$} & 0,4215 & 0,3156 & 1,5413 & 1,0597 & 1,9000 & 1,2413 \\
\hline 6 & $\begin{array}{l}0 \\
1 \\
1 \\
2 \\
2 \\
3 \\
3 \\
3 \\
4 \\
4 \\
5 \\
5 \\
6 \\
7\end{array}$ & $\begin{array}{l}6 \\
5 \\
7 \\
4 \\
6 \\
3 \\
5 \\
7 \\
4 \\
6 \\
5 \\
7 \\
6 \\
7\end{array}$ & $\begin{array}{l}{[0]} \\
{[0,0139]} \\
{[0]} \\
0,0615 \\
{[0]} \\
0,1528 \\
0 \\
{[0]} \\
0,0104 \\
{[0]} \\
0\end{array}$ & $\begin{array}{c}{[0]} \\
0,01030 \\
{[0]} \\
0,07089 \\
{[0]} \\
0,15289 \\
0 \\
{[0]} \\
0 \\
{[0]} \\
0\end{array}$ & $\begin{array}{c}{[0,0005]} \\
0,1346 \\
{[0,0002]} \\
0,2613 \\
{[0,01]} \\
0,4237 \\
0,0713 \\
{[0]} \\
0,0605 \\
{[0,0005]} \\
0,0008 \\
{[0]}\end{array}$ & $\begin{array}{c}{[0,0005]} \\
0,1177 \\
{[0,0002]} \\
0,28128 \\
{[0,005]} \\
0,42848 \\
0,04492 \\
{[0]} \\
0,06071 \\
{[0,0003]} \\
0,00074 \\
{[0]}\end{array}$ & $\begin{array}{c}{[0,003]} \\
0,4272 \\
{[0,005]} \\
0,3935 \\
{[0,06]} \\
0,2406 \\
0,1839 \\
{[0,004]} \\
0,1156 \\
{[0,007]} \\
0,0056 \\
{[0]}\end{array}$ & $\begin{array}{c}{[0,003]} \\
0 j 34461 \\
{[0,003]} \\
0,33297 \\
{[0,05]} \\
0,26157 \\
0,14125 \\
{[0,004]} \\
0,11758 \\
{[0,005]} \\
0,0059 \\
{[0]} \\
{[0]}\end{array}$ \\
\hline \multicolumn{3}{|c|}{$Q \mathrm{~L} / 10^{-2} a_{0}^{2}:$} & 0,2386 & 0,2341 & 0,9634 & 0,9398 & 1,4454 & 1,2698 \\
\hline 7 & $\begin{array}{l}0 \\
1 \\
2 \\
2 \\
3 \\
3 \\
4 \\
4 \\
5 \\
6\end{array}$ & $\begin{array}{l}7 \\
6 \\
5 \\
7 \\
4 \\
6 \\
5 \\
7 \\
6 \\
7\end{array}$ & $\begin{array}{l}{[0]} \\
{[0,0016]} \\
{[0,024]} \\
{[0]} \\
{[0,06]} \\
{[0]} \\
0,0008\end{array}$ & $\begin{array}{l}{[0]} \\
{[0]} \\
{[0,02]} \\
{[0]} \\
{[0,06]} \\
{[0]}\end{array}$ & $\begin{array}{c}{[0]} \\
{[0,048]} \\
0,0636 \\
{[0]} \\
0,3640 \\
{[0,02]} \\
0,0372 \\
{[0]} \\
{[0]}\end{array}$ & $\begin{array}{c}{[0]} \\
{[0,048]} \\
0,07776 \\
{[0]} \\
0,23719 \\
{[0,02]} \\
0,01995 \\
{[0]} \\
{[0]}\end{array}$ & $\begin{array}{c}{[0]} \\
{[0,20]} \\
0,1032 \\
{[0,03]} \\
0,3196 \\
{[0,05]} \\
0,1121 \\
{[0]} \\
{[0,005]} \\
{[0]}\end{array}$ & $\begin{array}{l}{[0]} \\
{[0,20]} \\
0,17757 \\
{[0,02]} \\
0,20534 \\
{[0,04]} \\
0,06097 \\
{[0]} \\
{[0,005]} \\
{[0]}\end{array}$ \\
\hline \multicolumn{3}{|c|}{$Q \mathrm{~L} / 10^{-2} a_{0}^{2}:$} & 0,0864 & 0,08 & 0,5328 & 0,4029 & 0,8199 & 0,7089 \\
\hline 8 & $\begin{array}{l}0 \\
1 \\
2 \\
3 \\
3 \\
4 \\
4 \\
5 \\
5 \\
6 \\
7\end{array}$ & $\begin{array}{l}8 \\
7 \\
6 \\
5 \\
7 \\
4 \\
6 \\
5 \\
7 \\
6 \\
7\end{array}$ & $\begin{array}{l}{[0]} \\
{[0]} \\
{[0,009]} \\
{[0,011]} \\
{[0]} \\
{[0]}\end{array}$ & $\begin{array}{l}{[0]} \\
{[0]} \\
{[0,009]} \\
{[0,011]} \\
{[0]} \\
{[0]}\end{array}$ & $\begin{array}{l}{[0]} \\
{[0,0136]} \\
{[0,010]} \\
0,1311 \\
{[0,004]} \\
0,0731 \\
{[0,007]} \\
0,0057 \\
{[0]} \\
{[0]}\end{array}$ & $\begin{array}{l}{[0]} \\
{[0,0136]} \\
{[0,009]} \\
0,11132 \\
{[0,003]} \\
{[0,07487]} \\
{[0,005]} \\
0,00574 \\
{[0]} \\
{[0]}\end{array}$ & $*$ & $*$ \\
\hline \multicolumn{3}{|c|}{$Q \mathrm{~L} / 10^{-2} a_{0}^{2}:$} & 0,02 & 0,02 & 0,2445 & 0,22117 & & \\
\hline
\end{tabular}

* Hier schien die Abschätzung für $l_{1}, l_{2}>5$ so unsicher, daß darauf verzichtet wurde und lieber der Gesamtwert $Q_{\mathrm{L}}$ für $L=8$ in Tab. 3 zusammen mit den Beiträgen für $L>8$ abgeschätzt wurde.

Tab. 2. Fortsetzung. 


\begin{tabular}{|c|c|c|c|c|c|c|}
\hline \multirow{2}{*}{$L$} & \multicolumn{2}{|c|}{$k_{0}^{2}=9$ at $\varepsilon$} & \multicolumn{2}{c|}{$k_{0}^{2}=12$ at $\varepsilon$} & \multicolumn{2}{c|}{$k_{0}^{2}=20$ at $\varepsilon$} \\
\cline { 2 - 7 } & ohne Austausch & mit Austausch & ohne Austausch & mit Austausch & ohne Austausch & mit Austaurch \\
\hline 0 & 0,0888 & 0,0888 & 0,2350 & 0,2395 & 0,2049 & 0,2267 \\
1 & 0,3806 & 0,2733 & 1,1838 & 0,8331 & 1,1788 & 0,8254 \\
2 & 0,6576 & 0,4280 & 1,9926 & 1,3140 & 1,8585 & 1,3231 \\
3 & 0,3626 & 0,2266 & 1,2350 & 0,7891 & 1,4909 & 0,9967 \\
4 & 0,7184 & 0,5818 & 2,1693 & 1,7055 & 1,9469 & 1,5271 \\
5 & 0,4215 & 0,3156 & 1,5413 & 1,0597 & 1,9000 & 1,2413 \\
6 & 0,2386 & 0,2341 & 0,9634 & 0,9398 & 1,4454 & 1,2689 \\
7 & 0,0864 & 0,080 & 0,5328 & 0,4029 & 0,8199 & 0,7089 \\
8 & 0,020 & 0,020 & 0,2445 & 0,2212 & {$[2,27]$} & {$[2,96]$} \\
\hline 8 & {$[0,021]$} & {$[0,025]$} & {$[0,33]$} & {$[0,27]$} & 13,1153 & 11,0781 \\
\hline $10^{-2} a_{0}^{2}$ & 2,9955 & 2,2732 & 10,4277 & 7,7748 & & 1 \\
\hline
\end{tabular}

Tab. 3. Beiträge $Q_{L}$ der einzelnen Gesamtdrehimpulse $L$ zum Gesamtionisationsquerschnitt $\left(10^{-2} a_{0}{ }^{2}\right)$.

zu niedrig angesetzt war. Für $L>8$ (bei $k_{0}^{2}=20$ für $L>7$ ) wurde angenommen, daß die Beiträge für gerades $L$ wie auch für ungerades $L$ entsprechend einer geometrischen Progression klein werden $\left(Q_{L+2} / Q_{L}=\right.$ const $)$.

Der Gesamtwirkungsquerschnitt ist in Tab. 4 und Abb. 4 verglichen mit Werten, die nach andern Formeln berechnet sind. Die Werte unter „Bons“ folgen aus einer Formel von Burhop ${ }^{20}$ für die Ionisation wasserstoffähnlicher 2s-Bahnen in Bonsscher Näherung. Die benutzten Parameter sind im Abschnitt Rechentechnik b) angegeben. Die „ElwerT“. Werte sind nach (4) bestimmt. GRyzinski ${ }^{21}$ gibt eine Verbesserung der klassischen Formel von Thomson, bei der die Bewegung der Atomelektronen berücksichtigt wird. Allerdings bleibt auch Gryzinski bei der Näherung des Zweier-Stoßes. FABre de LA RIPELle ${ }^{22}$ schlägt vor, den Wirkungsquerschnitt in der Form

$$
Q_{\text {red }}=a \cdot \ln U /(U+C)
$$

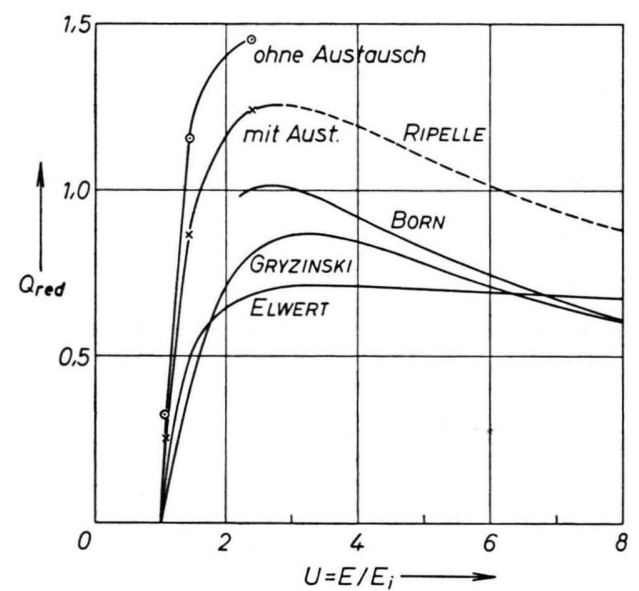

Abb. 4. Gesamtionisationsquerschnitt $Q_{\mathrm{red}}=Q\left(E_{\mathrm{i}} / h c \mathrm{Ry}\right)^{2} /$ $n \pi a_{0}{ }^{2}$, $\bigcirc$ hier berechnet ohne Austausch, $\mathrm{x}$ hier berechnet mit Austausch großer Reichweite. Die Kurve „RIPELLE“ ist angepaßt bei $k_{0}^{2}=20(U=2,39)$ und läuft innerhalb der Zeichengenauigkeit durch die von uns berechneten Punkte. $E_{\mathrm{i}}=8,372 \cdot 13,6 \mathrm{eV}, \quad n=2=$ Anzahl der gleichwertigen äußeren Elektronen.

\begin{tabular}{|c|c|c|c|c|c|c|c|c|c|}
\hline \multicolumn{2}{|c|}{$Q / a_{0}^{2}$} & \multicolumn{5}{c|}{$Q_{\text {red }}=Q\left(E_{\mathrm{i}} / 13,6 \mathrm{eV}\right)^{2} / 2 \pi a_{0}^{2}$} \\
\hline $\begin{array}{c}k_{0}^{2}=2 E \\
\text { (at.u.) }\end{array}$ & $U=E / E_{\mathrm{i}}$ & $\begin{array}{c}\text { hier ohne } \\
\text { Austausch }\end{array}$ & $\begin{array}{l}\text { hier mit } \\
\text { Austausch }\end{array}$ & $\begin{array}{c}\text { hier ohne } \\
\text { Austausch }\end{array}$ & $\begin{array}{c}\text { hier mit } \\
\text { Austausch }\end{array}$ & BoRN & ELwERT & GRYZINSKI & R RIPELLE \\
\hline 9 & 1,075 & 0,030 & 0,0227 & 0,335 & 0,253 & & 0,133 & 0,032 & 0,227 \\
12 & 1,433 & 0,104 & 0,0777 & 1,16 & 0,867 & & 0,477 & 0,381 & 0,856 \\
20 & 2,389 & 0,131 & 0,111 & 1,46 & 1,24 & 1,002 & 0,690 & 0,810 & 1,244 \\
25,116 & 3 & & & & & 1,004 & 0,711 & 0,862 & 1,250 \\
41,86 & 5 & & & & & 0,827 & 0,704 & 0,780 & 1,099 \\
66,98 & 8 & & & & & 0,608 & 0,678 & 0,607 & 0,887 \\
\hline
\end{tabular}

Tab. 4. Gesamt-Ionisationsquerschnitt $\left(E_{\mathrm{i}}=8,372 \cdot 13,6 \mathrm{eV}\right)$.

20 E. H. S. Burhop, Proc. Camb. Phil. Soc. 36, 43 [1940].

21 M. Gryzinski, Phys. Rev. 115, 374. [1959].
22 M. Fabre de la Ripelle, J. Phys. Rad. 10, 318 [1949]. 
anzusetzen mit 2 freien Parametern, $a, C$. Hier wurde $C=0$ gesetzt und mit $a=3,41$ Übereinstimmung für $k_{0}^{2}=20$ erreicht. Dann geht die Ripellesche Kurve innerhalb der Zeichengenauigkeit durch unsere gerechneten Punkte $k_{0}{ }^{2}=9$ und 12. Wegen des richtigen asymptotischen Verhaltens scheint uns die Form (37) zweckmäßig zur Darstellung des Wirkungsquerschnitts, sofern man z. B. Größe und Lage des Maximums von $Q$ kennt, um $a$ und $C$ festzulegen. Für unseren Fall ergibt sich mit $C=0$ der maximale Wirkungsquerschnitt zu

$$
Q_{\text {red, } \max }=a / e=1,25
$$

(e Basis der natürlichen Logarithmen),

$$
Q=0,112 a_{0}^{2}=3,15 \cdot 10^{-18} \mathrm{~cm}^{2}
$$

an der Stelle $\quad U=e=2,72$,

$$
E=22,8 h c \text { Ry }=310 \mathrm{eV} \text {. }
$$

Wie auch in Rechnungen anderer Autoren ${ }^{23}$ ergibt sich der Wirkungsquerschnitt um einen Faktor 2 bis 3 größer als nach der Elwertschen Formel. Burgess und Seaton führen diese Diskrepanz zum Teil darauf zurück, daß der reduzierte Wirkungsquerschnitt keine universale Funktion ist, sondern mit wachsender Ionenladung größer wird. Andererseits scheint es, als ob unsere Näherung, die jeweils nur den gerade interessierenden Vorgang in Betracht zieht, ohne die Möglichkeit anderer inelastischer Prozesse zu berücksichtigen, zu große Werte für den Wirkungsquerschnitt liefert. Um diesen Effekt abzuschätzen, ist geplant, den einen oder anderen Anregungsquerschnitt für $\mathrm{OV} \mathrm{zu}$ berechnen. Burgess, Seaton und van Regemorter ${ }^{24}$ schlagen eine Formel vor, die den Ionisationsquerschnitt bei Elektronenstoß verknüpft mit den Photoionisations-Querschnitten:

$$
Q(E)=\frac{158}{E / h c \mathrm{Ry}} \int_{0}^{E-E_{1}} \bar{g} a_{\nu}(W) \frac{\mathrm{d} W}{E_{\mathrm{i}}+W} .
$$

$a_{v}(W)$ ist der Photoionisations-Querschnitt, $E / h c$ Ry ist die kinetische Energie des stoßenden Elektrons in Rydberg-Einheiten $(h c \mathrm{Ry}=13,6 \mathrm{eV}) . W$ ist die kinetische Energie des losgeschlagenen Elektrons ( $W / h c \mathrm{Ry}=k_{2}{ }^{2} /$ at. u. in der Bezeichnung unserer Gleichungen), $\bar{g}$ ist ein GaUnT-Faktor, der zwischen 0,1 und 1 liegen kann, für den die genannten Autoren noch genauere Angaben machen. Die Formel (38) beruht auf der Annahme, daß der Elektronen-

23 M. J. Seaton und Mitarbeiter, persönliche Mitteilung.
stoß-Querschnitt im wesentlichen durch die optisch erlaubten Übergänge bestimmt ist. Benutzen wir unsere Ergebnisse, um rückwärts aus der Formel oben den Gaunt-Faktor $\bar{g}$ für $\mathrm{O} \mathrm{V}$ zu bestimmen, so ergibt sich näherungsweise für $k_{0}{ }^{2}=12$ nach SeAtoN $\left(E_{\mathrm{i}}=8,372 h c \mathrm{Ry} ; E-E_{\mathrm{i}}=3,63 h c \mathrm{Ry}\right)$ :

$$
\begin{aligned}
a_{v_{\mathrm{o}}}=a_{\nu}(0) & =0,77 \cdot 10^{-18} \mathrm{~cm}^{2} \\
& =0,0275 a_{0}^{2} \quad \text { (Schwelle), }
\end{aligned}
$$

$a_{v}(1,81 h c \mathrm{Ry}) \approx 0,73 a_{v_{0}} \quad$ (Mitte des Integrationsintervalls), $a_{v}(3,63 h c \mathrm{Ry}) \approx 0,55 a_{v_{0}} \quad\left(W=E-E_{\mathrm{i}}\right)$, daraus

\begin{tabular}{|c|c|c|c|c|}
\hline & Schwelle & $\begin{array}{c}\text { Mitte } \\
\text { d. Inter- } \\
\text { valles }\end{array}$ & $\begin{array}{c}W= \\
E-E_{\mathrm{i}}\end{array}$ & $\begin{array}{c}\text { Mittel- } \\
\text { wert }\end{array}$ \\
\hline$\frac{a_{\nu}(W)}{a_{0}^{2}\left(E_{\mathrm{i}}+W\right)}$ & 0,00328 & & 0,00126 & 0,00207 \\
\hline
\end{tabular}

und

$$
\frac{Q(E)}{a_{0}{ }^{2}} \approx \frac{158}{12} \bar{g} \cdot 0,00207 \cdot(12-8,372)=0,10 \bar{g} .
$$

Man erhält nach Tab. $3 \quad \bar{g}= \begin{cases}1,0 & \text { ohne Austausch, } \\ 0,78 \text { mit Austausch. }\end{cases}$ Aus den optischen Übergängen allein würde folgen (Tab. 1) :

$$
Q\left(E, l_{2}=1\right) / a_{0}{ }^{2}=0,017, \quad \bar{g}=0,17 .
$$

Nach den genaueren Angaben, die Burgess, Seaton und van REgemorter bezüglich $\bar{g}$ machen, sollte für $\mathrm{OV} \bar{g} \approx 1$ sein. Die Übereinstimmung ist besser als man erwarten konnte in einem Fall, bei dem die optischen Übergänge eine so geringe Rolle spielen.

\section{Rechentechnik}

a) Die Winkelkoeffizienten $c_{l_{2}, l_{1}}^{L}$, definiert in (30), haben wir aus den von Condon und Shortley angegebenen Formeln berechnet. Es ergibt sich

$c_{l_{2}, l_{1}}^{L}=(-1)^{1 / 2\left(-L+l_{1}+l_{2}\right)} \sqrt{\frac{2 l_{1}+1}{2 l_{2}+1}} f\left(L, l_{1}, l_{2}\right)$,

wobei $f$ eine symmetrische Funktion seiner Argumente ist und der Faktor davor im Zähler den Drehimpuls des gestreuten, im Nenner den des losgeschlagenen Elektrons enthält. Dabei ist

$$
f^{2}\left(l_{0}, l_{1}, l_{2}\right)=\frac{g\left(d_{0}\right) g\left(d_{1}\right) g\left(d_{2}\right)}{\left(l_{0}+l_{1}+l_{2}+1\right) g(s)}
$$

24 A. Burgess, M. J. Seaton u. H. van Regemorter, Proc. Phys. Soc., Lond., im Druck. 
mit $s=\frac{1}{2}\left(l_{0}+l_{1}+l_{2}\right), \quad d_{i}=s-l_{i}, \quad g(n)=\frac{(2 n) !}{n !^{2}}$.

b) Die gebundenen Wellenfunktionen $\varphi_{0}=2 \mathrm{~s}$ Funktion für den Grundzustand von $\mathrm{OV}$ und $\varphi_{1}=2$ s-Funktion für O VI wurden einer Arbeit von Roothaan ${ }^{25}$ entnommen, der den Ansatz

$$
\varphi=r \sum_{n}\left(A_{n}+r B_{n}\right) e^{-a_{n} r}
$$

einem Hartree-Fock-Verfahren unterwirft. Er erhält (in atomaren Einheiten)

\begin{tabular}{|c|c|c|c|c|c|}
\hline \multicolumn{3}{|c|}{$0 \mathrm{~V}\left(\varphi_{0}\right)$} & \multicolumn{3}{c|}{$0 \mathrm{VI}\left(\varphi_{1}\right)$} \\
\hline$\alpha_{n}$ & $A_{n}$ & $B_{n}$ & $\alpha_{n}$ & $A_{n}$ & $B_{n}$ \\
\hline 12,5 & $-5,858$ & $-10,799$ & 12,5 & $-4,269$ & $-7,846$ \\
7,4 & $-4,796$ & $-26,477$ & 7,4 & $-2,346$ & $-11,896$ \\
2,94 & $-1,300$ & $+20,467$ & 3,22 & $-6,068$ & $+31,121$ \\
\hline
\end{tabular}

Diese Funktionen sind numerisch berechneten HARTREe-Fock-Funktionen gleichwertig und lassen sich besser für beliebige Argumentwerte bestimmen.

Zur Berechnung der BonN-Näherung mußte $\varphi_{0}$ durch eine wasserstoffartige Wellenfunktion:

$$
\tilde{\varphi}_{0}=\text { const } \cdot r(1-\mu r) e^{-\mu r}
$$

ersetzt werden. Wir haben nach SLATER gesetzt: $\mu=3$.

c) Wahl der Variabeln und Schrittlängen. Um Änderungen in der Schrittlänge bei der Integration der Dgl. (16) und den Quadraturen (28) zu vermeiden, wurde eine Variable $\sigma$ gewählt, die sich für kleine $r$ wie $r^{1 / 4}$ verhielt und für große $r$ linear wurde, gemäß

$$
\mathrm{d} r=a \frac{\sigma^{3}}{1+\sigma^{3}} \mathrm{~d} \sigma .
$$

Die Schrittweite bestimmte sich daraus, daß auf eine Wellenlänge der $\chi$-Funktion mindestens 12 Punkte entfallen müssen:

$$
\begin{aligned}
& 12 \Delta r & \leq 2 \pi / k \\
\text { oder } & \Delta \sigma & \leq(2 k a)^{-1} .
\end{aligned}
$$

Wir haben $\quad a=10, \quad \Delta \sigma=2^{-7} \quad$ gewählt.

$$
\text { d) Die Dgl. (16) } \mathrm{d}^{2} \chi / \mathrm{d} r^{2}=v(r) \chi(r)
$$

wurde auf die Variable $\sigma$ transformiert nach dem allgemeinen Schema

$$
\mathrm{d} r=\mathrm{d} \sigma / g(\sigma), \quad \chi(r)=\zeta(\sigma) / \sqrt{g(\sigma)},
$$

25 C. C. J. Roothan, L. M. Sachs u. A. W. Weiss, Rev. Mod. Phys. 32, 186 [1960].

26 G. Stracke, Bahnbestimmung der Planeten und Kometen, Berlin 1929, S. 77.

27 E. Trefftz, A. Schlüter, K.-H. Detrimar u. K. Jörgens, Z. mit

$$
\mathrm{d}^{2} \zeta / \mathrm{d} \sigma^{2}=\varphi(\sigma) \zeta(\sigma),
$$

$$
\varphi(\sigma)=u(\sigma)+\frac{v(r(\sigma))}{g^{2}(\sigma)}, \quad u(\sigma)=\frac{1}{\sqrt{g}} \frac{\mathrm{d}^{2}}{\mathrm{~d} \sigma^{2}} \sqrt{g},
$$

Bei uns ist

$$
g(\sigma)=a^{-1}\left(1+\sigma^{-3}\right), \quad u(\sigma)=\frac{1}{\sigma^{2}\left(1+\sigma^{3}\right)}\left[6-\frac{9 / 4}{1+\sigma^{3}}\right] .
$$

Für die Integration der Differentialgleichung wurde ein Verfahren benutzt, das man als „aufsummiertes Numerov-Verfahren" 26 bezeichnen könnte und das sich bereits bei früheren Rechnungen ${ }^{27}$ bewährt hat:

Die Adams-Störmer-Formel ${ }^{28}$

$$
\begin{aligned}
\zeta_{\nu+1}-2 \zeta_{\nu}+\zeta_{\nu-1}= & h^{2} \varphi_{\nu} \zeta_{\nu}+\frac{h^{2}}{12} \\
& \cdot\left(\varphi_{\nu+1} \zeta_{\nu+1}-2 \varphi_{\nu} \zeta_{\nu}+\varphi_{\nu-1} \zeta_{\nu-1}\right)
\end{aligned}
$$

summiert man auf über alle $v$ bis $v=n-1$ und löst nach $\zeta_{n}$ auf:

$$
\begin{gathered}
\left(1-\frac{h^{2}}{12} \varphi_{n}\right) \zeta_{n}=\left(1-\frac{h^{2}}{12} \varphi_{n-1}\right) \zeta_{n-1}+h C_{n-1}, \\
h C_{n}=h C_{n-1}+h^{2} \varphi_{n} \zeta_{n} .
\end{gathered}
$$

Aus zwei Anfangswerten $\zeta_{m-1}, \zeta_{m}$, die in unserem Fall aus einer TAYLOR-Entwicklung für $\chi(r)$ gewonnen werden, ergibt sich der Wert $C_{m-1}$, und das Verfahren kann beginnen. Bei dieser aufsummierten Methode sind gegenüber dem Adams-Störmer- oder Numerov-Verfahren die Abrundungsfehler sehr viel kleiner. Bei gegebenem $h^{2} \varphi$ sind pro Schritt 2 Multiplikationen und 1 Division nötig.

Schließlich muß für $\sigma \rightarrow \infty$ die Amplitude der Funktion $\zeta(\sigma)$ bestimmt werden. Dazu wurde ein Verfahren von Rudкјøвing ${ }^{29}$ benutzt. Aus (16) und (R 7-12) folgt

$$
\varphi(\sigma) \rightarrow-a^{2} k^{2} \quad \text { für } \quad \sigma \rightarrow \infty .
$$

Der Ansatz $\zeta(\sigma)=A(\sigma) \cos b(\sigma)$,

$$
z(\sigma)=\mathrm{d} b / \mathrm{d} \sigma
$$

führt, eingesetzt in (R 10), zu

$$
\begin{aligned}
& A(\sigma)=C z^{-1 / 2}(\sigma), \\
& z^{2}=-\varphi(\sigma)+A^{\prime \prime} \mid A=-\varphi(\sigma)+z^{1 / 2} \frac{\mathrm{d}^{2}}{\mathrm{~d} \sigma^{2}}\left(z^{-1 / 2}\right) .
\end{aligned}
$$

Astrophys. 44, 1 [1957]. - F. B. Malik u. E. Treffiz, Z. Astrophys. 50, 96 [1960].

28 G. Schulz, Formelsammlung zur praktischen Mathem., VI, § 3, Samml. Göschen 1937.

29 M. Rudкјøвing, Kg. Danske Vid. Selsk., Mat. fys. Medd. 18, Nr. 2 (1940). 
$z$ wird iterativ bestimmt, beginnend mit $z_{0}=(-\varphi)^{1 / 2}$. Die erste Näherung ist im allgemeinen genau genug. Daraus wird $b(\sigma)$ über ein passendes Intervall integriert:

$$
b_{2}-b_{1}=\int_{\sigma_{1}}^{\sigma_{2}} z \mathrm{~d} \sigma .
$$

Es soll $b_{2}-b_{1} \lesssim \pi / 2$ sein. Mit der Bezeichnung

$$
\begin{aligned}
& A\left(\sigma_{1}\right) \sqrt{z\left(\sigma_{1}\right)}=B_{1}, \\
& A\left(\sigma_{2}\right) \sqrt{z\left(\sigma_{2}\right)}=B_{2}
\end{aligned}
$$

erhält man die Konstante $C$

$$
C=\frac{\left[B_{1}{ }^{2}+B_{2}{ }^{2}-2 B_{1} B_{2} \cos \left(b_{2}-b_{1}\right)\right]^{1 / 2}}{\sin \left(b_{2}-b_{1}\right)} .
$$

Die Funktion $\chi(r)$, die der Bedingung (19b) genügt, ergibt sich aus dem (mit beliebiger Anfangssteigung berechneten) $\zeta$ zu

$$
\chi(r(\sigma))=\zeta(\sigma) / C \cdot \sqrt{g(\sigma)} .
$$

e) Die Integrale

$$
\begin{gathered}
V=\int \chi_{1}\left(r_{1}\right) \chi_{2}\left(r_{2}\right) \frac{r_{<}^{l}}{r_{>}^{l+1}} \chi_{0}\left(r_{1}\right) \varphi_{0}\left(r_{2}\right) \mathrm{d} r_{1} \mathrm{~d} r_{2} \\
=\int_{0}^{\infty} \chi_{1} \chi_{0} r^{-l-1} \int_{0}^{r} \chi_{2} \varphi_{0} r^{\prime l} \mathrm{~d} r^{\prime} \mathrm{d} r \quad(\mathrm{R} 21) \\
+\int_{0}^{\infty} \chi_{2} \varphi_{0} r^{-l-1} \int_{0}^{r} \chi_{1} \chi_{0} r^{\prime l} \mathrm{~d} r^{\prime} \mathrm{d} r
\end{gathered}
$$

wurden in Richtung wachsender $r$ integriert, zunächst mit Hilfe der TAYLOR-Entwicklung, dann numerisch, das innere Integral mit einer 4-Punkte-Formel, das äußere durch Aufsummieren der Ordinaten (alles in der Variabeln $\sigma$ ) bis zu einem Radius

$$
R \approx 12,
$$

bei dem die Atomfunktion $\varphi_{0}$ klein genug geworden ist. Für die Intervallgrenzen des äußeren Integrals muß eine Korrektur angebracht werden, die der Genauigkeit der 4-Punkte-Formel entspricht. Das Außen-Integral

$$
\int_{R}^{\infty} \chi_{0} \chi_{1} r^{-l-1} \mathrm{~d} r
$$

kann mit Hilfe der Differentialgleichungen für $\chi_{0}, \chi_{1}$

$$
\left(\frac{\mathrm{d}^{2}}{\mathrm{~d} r^{2}}-\frac{l_{i}\left(l_{i}+1\right)}{r^{2}}+\frac{2 Z_{i}}{r}+k_{i}^{2}\right) \chi_{i}=0
$$

durch eine semikonvergente Reihe ausgedrückt werden. Es sei

$$
\begin{aligned}
E_{-} & =k_{0}^{2}-k_{1}^{2}, \\
E_{+} & =k_{0}^{2}+k_{1}^{2}, \\
2 Z_{+} & =2\left(Z_{1}+Z_{0}\right)=18, \\
2 Z_{-} & =2\left(Z_{1}-Z_{0}\right)=2, \\
A_{+} & =l_{1}\left(l_{1}+1\right)+l_{0}\left(l_{0}+1\right), \\
\Lambda_{-} & =l_{1}\left(l_{1}+1\right)-l_{0}\left(l_{0}+1\right) .
\end{aligned}
$$

Dann ist

$$
\begin{aligned}
\int_{R}^{\infty} \chi_{0} \chi_{1} r^{-n} \mathrm{~d} r=\frac{1}{E_{-} R^{n}}\{ & \left\{( \chi _ { 1 } \frac { \mathrm { d } \chi _ { 0 } } { \mathrm { d } r } - \chi _ { 0 } \frac { \mathrm { d } \chi _ { 1 } } { \mathrm { d } r } ) \left[1+\frac{1}{E_{-}}\left(\frac{2 Z_{-}}{R}-\frac{\Lambda_{-}}{R^{2}}\right)\right.\right. \\
& +\frac{1}{E_{-}^{2}}\left(\frac{-2 n(n+1) E_{+}+4 Z_{-}^{2}}{R^{2}}+\frac{-n(2 n+3) 2 Z_{+}-4 Z_{-} \Lambda_{-}}{R^{3}}\right) \\
& \left.+\frac{1}{E_{-}^{3}} \frac{-2\left(3 n^{2}+6 n+2\right) E_{+} \cdot 2 Z_{-}+8 Z_{-}^{3}}{R^{3}}\right] \\
& +\left(\chi_{1} \frac{\mathrm{d} \chi_{0}}{\mathrm{~d} r}+\chi_{0} \frac{\mathrm{d} \chi_{1}}{\mathrm{~d} r}\right)\left[\frac{n(n+1)}{E_{-} R^{2}}+\frac{(n+2)(2 n+1) 2 Z_{-}}{E_{-}^{2} R^{3}}\right] \\
& +\left(\chi_{1} \chi_{0} E_{+}+2 \frac{\mathrm{d} \chi_{1}}{\mathrm{~d} r} \frac{\mathrm{d} \chi_{0}}{\mathrm{~d} r}\right)\left[\frac{n}{E_{-} R}+\frac{1}{E_{-}^{2}}\left(\frac{(2 n+1) 2 Z_{-}}{R^{2}}-\frac{2(n+1) \Lambda_{-}}{R^{3}}\right) \quad(\mathrm{R} 24)\right. \\
& \left.+\frac{1}{E_{-}^{3}} \frac{-2 n(n+1)(n+2) E_{+}+3(n+1) \cdot 4 Z_{-}^{2}}{R^{3}}\right] \\
& \left.+\left(\chi_{1} \chi_{0}\right)\left[\frac{1}{E_{-}}\left(\frac{n 2 Z_{+}}{R^{2}}+\frac{n(n+1)(n+2)-n \Lambda_{+}}{R^{3}}\right)+\frac{(2 n+1) 4 Z_{+} Z_{-}}{E_{-}^{2} R^{3}}\right]+\ldots\right\} .
\end{aligned}
$$

$\chi, \mathrm{d} \chi / \mathrm{d} r$ sind an der Stelle $R$ zu nehmen.

f) Für das Integral über $k_{2}^{2}$ wurde die Gausssche Quadraturformel benutzt, und zwar für $k_{0}^{2}=9$ mit 4 Stützpunkten, für $k_{0}^{2}=12$ und 20 mit 6 Stützpunkten.
Die Rechnungen wurden auf der IBM 704-Maschine in Paris durchgeführt. Die Berechnung des Gesamtwirkungsquerschnitts für einen Energiewert $k_{0}{ }^{2}$ dauerte 1 Stunde.

Wir möchten Herrn Professor Biermann sowie unseren Mitarbeitern am hiesigen Institut für ihr Interesse 
an dieser Arbeit danken. Sehr zu Dank verbunden sind wir auch den Kollegen M. J. Seaton und A. Burgess vom University College of London für aufschlußreiche Diskussionen. Der IBM danken wir für die kostenlose Úberlassung von Rechenzeit an der Pariser Maschine 704, durch die die Arbeit erst ermöglicht wurde. Ins- besondere hat uns die stete Hilfsbereitschaft der Herren Dr. Everling, Mürle, Trefz und Endrees von IBM viel Mühe erspart. Der hiesigen Rechengruppe sei für ihre Hilfe bei der Vorbereitung der Rechnung sowie bei der Zusammenstellung der Tabellen und Abbildungen bestens gedankt.

\title{
Die Bedeutung des Energievergleidhes für die Güte einer Näherungslösung
}

\author{
Von H. Preuss \\ Aus dem Max-Planck-Institut für Physik und Astrophysik, München \\ (Z. Naturforschg. 16 a, 598-602 [1961] ; eingegangen am 22. Februar 1961)
}

\begin{abstract}
Es werden die verschiedenen Güteteste an Näherungsansätzen diskutiert und gezeigt, daß der Energievergleich kein ausreichendes Kriterium für die Güte einer Wellenfunktion ist. Unter Verwendung der lokalen Energie wird ein Variationsverfahren hergeleitet, welches mit einer allgemeineren Gütedefinition zusammenhängt und in erster Näherung bessere Erwartungswerte zu liefern vermag als dies mit Hilfe der Energievariation möglich ist.
\end{abstract}

Die meisten Berechnungen der Wellenfunktionen von Atomen und Molekülen basieren auf dem Energieminimumprinzip, in welchem die normiert angesetzten Näherungen $\tilde{\Psi} *$ der Eigenfunktionen $\Psi$ so variiert werden, daß der Erwartungswert der Energie $\langle\tilde{\Psi}|H| \tilde{\Psi}\rangle$ minimal wird.

$$
\langle\tilde{\Psi}|H| \tilde{\Psi}\rangle=\langle H\rangle=\min .
$$

In fast allen Fällen sind die Eigenfunktionen nicht bekannt. Man ist daher auf das Verfahren (1) angewiesen, in welchem eine endliche Anzahl von Parametern im Näherungsansatz Verwendung finden. Die auf diese Weise erhaltenen Energiewerte sind obere Grenzen für den Energieeigenwert des Grundzustan$\operatorname{des} E_{0}$

$$
\langle H\rangle-E_{0}=\varepsilon_{0}{ }^{2} \geqq 0, \quad \begin{aligned}
& \text { (Index } 0 \text { bedeutet } \\
& \text { Grundzustand) }
\end{aligned}
$$

und die entsprechenden Approximationen $\tilde{\Psi}$ stellen häufig keine ausreichenden Näherungen für die Eigenfunktionen dar. Man erkennt das daran, daß die mit $\tilde{\Psi}$ berechneten Erwartungswerte und Übergangselemente, die uns Informationen über ein vorliegendes System liefern sollen, mit den korrespondierenden Meßwerten, soweit diese bekannt sind, in vielen Fällen mangelhaft übereinstimmen.

Diese allgemein bekannte Tatsache ist oft schon deswegen hervorgehoben worden, weil die zu diesen

* Im folgenden als reell vorausgesetzt.
$\tilde{\Psi}$-Funktionen gehörenden $\varepsilon^{2} / E$-Werte nach (2) schon sehr klein sind und z. B. bei den konventionellen Verfahren (self-consistent-field-Verfahren, Konfigurationswechselwirkung) in der Größenordnung von einigen Prozent und kleiner gefunden werden.

Nur bei Verwendung einer sehr großen Anzahl von Parametern im Variationsansatz (beim Helium sind über 1000 verwendet worden ${ }^{1}$ ) kann auch in den mit $\tilde{\Psi}$ berechneten Erwartungswerten und Übergangselementen (Oszillationsstärken, Suszeptibilitäten, Elektronendichten am Kern usw.) weitgehende Übereinstimmung (kleiner als $1 \%$ Fehler) mit den exakten Werten erreicht werden.

Es erhebt sich also die Frage, inwieweit man berechtigt ist, aus der Größenordnung von $\varepsilon^{2}$ auf die Genauigkeit der Erwartungswerte und Übergangselemente zu schließen, die mit dem jeweiligen Näherungsansatz berechnet worden sind. Bevor wir auf diese Frage näher eingehen, müssen wir die bisher bekannten Kriterien für die Güte einer Variationsfunktion diskutieren.

\section{Die verschiedenen Kriterien}

Hier ist zu unterscheiden zwischen Relationen, die einen Vergleich zur exakten Lösung darstellen oder zumindestens diese beim Vergleich auf irgendeine

1 T. Kinoshita, Phys. Rev. 115, 366 [1959]. - C. L. Pekeris, Phys. Rev. 115, 1216 [1959]. 